\title{
Learning induces coordinated neuronal plasticity of metabolic demands and functional brain networks
}

\author{
Sebastian Klug ${ }^{1 \#, ~ G o d b e r ~ M ~ G o d b e r s e n ~}{ }^{1 \#}$, Lucas Rischka1, Wolfgang Wadsak ${ }^{2,3}$, Verena \\ Pichler $^{2,4}$, Manfred Klöbl ${ }^{1}$, Marcus Hacker ${ }^{2}$, Rupert Lanzenberger ${ }^{1}$, Andreas Hahn ${ }^{1 *}$ \\ ${ }^{1}$ Department of Psychiatry and Psychotherapy, Medical University of Vienna, Austria \\ ${ }^{2}$ Department of Biomedical Imaging and Image-guided Therapy, Division of Nuclear \\ Medicine, Medical University of Vienna, Austria \\ ${ }^{3}$ Center for Biomarker Research in Medicine (CBmed), Graz, Austria \\ ${ }^{4}$ Department of Pharmaceutical Sciences, Division of Pharmaceutical Chemistry, University \\ of Vienna, Austria
}

\begin{tabular}{|c|c|c|}
\hline Word count & 221 & Abstract \\
\hline & 4390 & Main section \\
\hline & 4146 & Methods \\
\hline \multicolumn{2}{|c|}{ Figures: 6, Tables: 0, References: 97} \\
Supplement: 2 figures \\
\hline
\end{tabular}

\# contributed equally

* Correspondence to: $\quad$ Andreas Hahn, A/Prof. PD PhD MSc

Email: andreas.hahn@meduniwien.ac.at

ORCID: 0000-0001-9727-7580

Department of Psychiatry and Psychotherapy

Medical University of Vienna, Austria

Waehringer Guertel 18-20, 1090 Vienna, Austria 


\section{ORCID}

Klug Sebastian

https://orcid.org/0000-0001-8714-6608

Godbersen Godber Mathis https://orcid.org/0000-0002-9739-0724

Rischka Lucas

https://orcid.org/0000-0002-6766-857X

Wadsak Wolfgang

https://orcid.org/0000-0003-4479-8053

Pichler Verena

https://orcid.org/0000-0003-4544-2438

Klöbl Manfred

https://orcid.org/0000-0003-2107-8803

Hacker Marcus

https://orcid.org/0000-0002-4222-4083

Lanzenberger Rupert

https://orcid.org/0000-0003-4641-9539

Hahn Andreas

https://orcid.org/0000-0001-9727-7580

\section{Clinical trials identifier: NCT03485066}

Ethics approval: ethics committee of the Medical University of Vienna (1479/2015)

Key words: neuroplasticity, simultaneous PET/MRI, brain metabolism, functional PET, functional connectivity, metabolic connectivity mapping 


\section{ABSTRACT}

The neurobiological basis of learning is reflected in adaptations of brain structure, network organization and energy metabolism. However, it is still unknown how different neuroplastic mechanisms act together and if cognitive advancements relate to general or task-specific changes. To address these questions, we tested how hierarchical network interactions contribute to improvements in the performance of a visuo-spatial processing task by employing simultaneous PET/MR neuroimaging before and after a 4-week learning period. We combined functional PET with metabolic connectivity mapping (MCM) to infer directional interactions across brain regions and subsequently performed simulations to disentangle the role of functional network dynamics and glucose metabolism. As a result, learning altered the topdown regulation of the salience network onto the occipital cortex, with increases in MCM at resting-state and decreases during task execution. Accordingly, a higher divergence between resting-state and task-specific effects was associated with better cognitive performance, indicating that these adaptations are complementary and both required for successful skill learning. Simulations further showed that changes at resting-state were dependent on glucose metabolism, whereas those during task performance were driven by functional connectivity between salience and visual networks. Referring to previous work, we suggest that learning establishes a metabolically expensive skill engram at rest, whose retrieval serves for efficient task execution by minimizing prediction errors between neuronal representations of brain regions on different hierarchical levels. 


\section{INTRODUCTION}

Learning is a fundamental cognitive process that allows an organism to adapt to its environment throughout the entire lifetime. As neuroscientific research has moved beyond the behavioral level to examine the underlying neurobiological mechanisms, numerous structural, functional, and molecular aspects of learning have been demonstrated.

Human magnetic resonance imaging (MRI) studies have revealed learning-induced neuroplastic changes in gray and white matter structure (Zatorre et al., 2012) as well as in functional networks that undergo dynamic reconfigurations (Bassett \& Mattar, 2017). These consistently showed that interactions of higher-order brain networks of cognitive control, such as cingulo-opercular, salience and fronto-parietal networks, among each other (Mohr et al., 2016) and with lower-level visual areas (Lewis et al., 2009) predict learning-related gain in task efficiency (Bassett et al., 2011). On the other hand, positron emission tomography (PET) imaging with the radiolabeled glucose analogue $\left[{ }^{18} \mathrm{~F}\right] \mathrm{FDG}$ further showed that brain areas involved in a visuo-spatial task performance also undergo metabolic adaptations after learning, indicating a more cost-effective use of metabolic resources (Haier et al., 1992).

However, most of the previous work only employed a single imaging modality at the same time, thus impeding to draw conclusions about the learning-induced relationships across different parameters of brain function. In addition, the effects of learning were investigated either in a general manner at resting state (e.g., gray and white matter structure (Zatorre et al., 2012), network adaptations) or specifically during task execution (e.g., metabolic demands (Haier et al., 1992), neuronal activation), while the direct comparison between both aspects largely remains missing (Cole et al., 2014). In sum, it is not clear whether intrinsic resting-state or taskrelated effects drive the improvement in cognitive performance after learning. Furthermore, the interaction between different indices of brain function and network adaptations is poorly understood. 
The framework of metabolic connectivity mapping (MCM) combines MRI-derived functional connectivity and glucose metabolism obtained with $\left[{ }^{18} \mathrm{~F}\right] \mathrm{FDG}$ PET, thereby enabling the computation of directional connectivity (Riedl et al., 2016). The underlying rationale is that the integration of metabolic information identifies the target region of a connection, since the majority of energy demands emerge post-synaptically (Attwell \& Laughlin, 2001; Harris et al., 2012; Mergenthaler et al., 2013). The two imaging parameters are also tightly linked on a physiological basis through glutamate-mediated processes that occur upon neuronal activation. Glutamate release increases cerebral blood flow via neurovascular coupling (Attwell et al., 2010; Mishra et al., 2016), which in turn affects the blood oxygen level dependent (BOLD) signal used for the assessment of functional connectivity. On the other hand, glutamate release also triggers glucose uptake into neurons (Lundgaard et al., 2015) and astrocytes (Zimmer et al., 2017), to meet increased energy demands for the reversal of ion gradients (Raichle \& Mintun, 2006; Harris et al., 2012; Magistretti \& Allaman, 2015). MCM thus constitutes a validated framework to investigate the associations of glucose metabolism and functional connectivity and decipher hierarchical interactions across brain regions by assigning directionality to connections (Hahn et al., 2020; Riedl et al., 2016). Furthermore, the use of functional PET (fPET) imaging allows to investigate metabolic demands at rest and during task execution in a single measurement (Hahn et al., 2016).

Combining these methodological advancements we have recently demonstrated that cognitive task performance strengthened the interplay of functional connectivity and glucose metabolism, specifically for feedforward connections to higher-order cognitive processing areas (Hahn et al., 2020). These data indicated that most of the metabolic cost originates from the switch of the resting-state to the task-related network interactions, which extended previous work showing that acute task performance itself leads to pronounced functional network reorganizations (Hearne et al., 2017) and increases in metabolic demands (Phelps et al., 1981). However, the corresponding effects induced by prolonged training of a task remain unknown. 
To address the open questions outlined above, we investigated learning-induced neuronal adaptations in functional brain networks and the underlying energy demands before and after healthy volunteers practiced a challenging visuo-spatial task for 4 weeks. Multimodal data analysis of simultaneously acquired PET/MRI data allowed us i) to assess the interaction of training-induced changes between functional connectivity and glucose metabolism, ii) to disentangle the neurobiological contributions of resting-state and task-specific effects that drive improvements in cognitive performance and iii) to reveal the hierarchical interplay across brain regions involved in the learning process. Since previous work has demonstrated the convergence of functional connectivity and glucose metabolism already during the first execution of a novel task (Hahn et al., 2020) we expect that after continuous skill learning this task-specific association is consolidated also at resting-state. We hypothesize that training effects will be further reflected in the interaction of higher-order brain regions involved in cognitive control. 


\section{RESULTS}

Multimodal brain imaging data and behavioral performance were acquired for 41 healthy participants in a longitudinal study design (figure 1). Subjects were assigned to either the training $(n=21)$ or passive control group $(n=20)$, which were carefully matched regarding the distributions of age, sex and general intelligence (all $p>0.5$ ). All subjects completed two PET/MRI scans approximately 4 weeks apart ( $p=0.2$ between groups) with simultaneous acquisition of fPET using $\left[{ }^{18} \mathrm{~F}\right] \mathrm{FDG}$, functional connectivity (blood oxygen level dependent signal (BOLD)) as well as $\mathrm{fMRI}$ (BOLD and arterial spin labeling (ASL), supplementary figure S1). Imaging was obtained at rest and during the performance of a cognitively challenging task (an adapted version of the video game Tetris $®$ ) at two levels of difficulty (easy, hard), which required rapid visuo-spatial processing, motor coordination and planning. Between the two PET/MRI measurements the training group practiced the cognitive task on a regular basis with the explicit aim to be able to manage the hard level at the second scan. Both PET/MRI sessions were accompanied by cognitive assessment to relate improvements in task performance to mental rotation, visual search or spatial planning.

First, we combined the imaging parameters of glucose metabolism (CMRGlu), blood flow $(\mathrm{CBF})$ and the BOLD signal for a functional delineation of brain regions with increased metabolic demands during task performance. In the next step, learning-induced changes in the networks encompassing the task-specific regions were investigated in the framework of MCM. MCM represents the associations between regional patterns of CMRGlu and BOLDderived functional connectivity (FC), thereby enabling inference on directional connectivity (Hahn et al., 2020; Riedl et al., 2016). For a thorough assessment of neuroplastic network effects, MCM was computed in an unbiased whole-brain approach for all three conditions (rest, easy and hard task). In addition, simulations were carried out to identify whether the metabolic underpinnings or network reorganizations drive the training-induced changes by manipulating spatial MCM correlations based on values of CMRGlu of FC. Finally, changes of gray matter volume and white matter microstructure were assessed to identify whether learning-induced 
adaptations of MCM are also mirrored by structural adaptations. Please see the supplement for a thorough methodological description.

\section{Learning-induced improvement in cognitive skill performance}

Task performance was given by score per minute achieved during Tetris®, which underlined the successful training (figure 2). At the first PET/MRI measurement (i.e., before the training period), the training and control group did not differ regarding task performance, neither for the easy nor the hard task condition (both $\mathrm{p}>0.5$ ). Subjects in the training group then practiced $53.6 \pm 5.2$ minutes per day for $20.9 \pm 1.5$ days within a 4 -week period using an online training program. This training period elicited significant changes in task performance (group*time* condition interaction, $p<10^{-5}$ ), with the training group performing significantly better in both task conditions after training. However, the improvement in task performance was particularly more pronounced for the hard task condition (group*time interaction, $p<10^{-9}$, figure $2 b)$ as compared to the easy condition ( $p<0.05$, figure $2 a)$, indicating a more robust learning effect in conjunction with higher task load and skill demand. In the training group, the task performance of the two PET/MRI scans also matched that obtained during the online training (figure 2c). Interestingly, task performance even further improved in the training group for the hard task level another 4 weeks after the second PET/MRI scan $(p<0.05$, figure $2 b)$, although no additional training was carried out. Furthermore, the training group $(p<0.001)$ but not the control group $(p>0.1)$ showed improved mental rotation performance after learning (group*time interaction $p<0.05$ ). A similar pattern was observed for the visual search task (interaction $p=0.05$, training group before vs. after learning $p=0.09$, control group $p>0.2$ ), but not for spatial planning performance (all $p>0.6$ ).

\section{Overlapping task-specific neuronal activation across imaging modalities}

To identify regional increases in neuronal activation elicited by task execution, we combined three imaging parameters that represent different indices of metabolic demands obtained during the first PET/MRI measurement. CMRGlu, CBF and BOLD signal changes during task 
execution (all $p<0.05$ FWE corrected) showed high spatial overlap in task-specific increases (Dice coefficient $=0.48-0.57$, supplementary figure S2), similar to our previous work(Hahn et al., 2020). Brain regions with mutual task-specific effects across imaging modalities (i.e., intersection) comprised the occipital cortex $\left(\mathrm{Occ}, 14.0 \mathrm{~cm}^{3}\right)$, intraparietal sulcus (IPS, $20.3 \mathrm{~cm}^{3}$ ) and frontal eye field $\left(\mathrm{FEF}, 6.9 \mathrm{~cm}^{3}\right)$, with the latter two representing the dorsal attention network (DAN). These areas served as target regions for the subsequent assessment of learninginduced changes with MCM. Two other clusters of overlapping task increases were observed in the ventral premotor cortex and occipital/temporal inferior cortex, which were however not further considered due to their limited spatial extent $\left(0.3\right.$ and $0.9 \mathrm{~cm}^{3}$, respectively).

\section{Learning-induced adaptations in metabolic connectivity mapping}

Proceeding from the above conjunction of metabolic demands during acute task execution, we investigated the corresponding network changes after practicing the same task over a 4-week training period by computing the association between CMRGlu and BOLD-derived FC. With Occ as the target region, learning-induced effects in MCM were observed in the dorsal anterior cingulate cortex (dACC) and the insula, both being integral parts of the salience network (SN, group ${ }^{*}$ time* condition interaction, all $p<0.05$ FWE-corrected, figure 3). This result was obtained independent of the contrast of interest, whereas two other regions (left insula and primary visual cortex) were observed just for one of the contrasts and will thus not be considered further. Post-hoc analysis showed that after the learning period MCM values of these two connections directed towards Occ increased at resting-state for the training group as compared to the control group (group*time interaction, all $\mathrm{p}<0.01$ ). In contrast, MCM of connections from dACC and insula decreased during execution of the hard task level $(p<0.01$ 0.05). Further analysis confirmed that these differences emerged exclusively from changes in the training group $(p<0.01-0.05)$ with no significant differences in the control group (all $p>0.09$ ). MCM values obtained at the first PET/MRI measurement were not significantly different for the two mentioned connections. Furthermore, no significant training-induced effects in MCM were 
observed for the other two regions that showed overlapping task-specific activation (FEF and IPS).

Based on the diverging training effects in the salience network (i.e., increased MCM at rest after training vs. decreased MCM during the task) we further tested whether the combination of these changes was related to cognitive performance. Indeed, the difference in MCM values between rest and the hard task for the connection from dACC to Occ was positively associated with the Tetris ${ }^{\circledR}$ score of the second PET/MRI scan $(\rho=0.46, p<0.05)$ and the area under the curve of scoring obtained during the entire 4-week training period $(\rho=0.56, p<0.01$, figure $4 a-$ b). MCM values of the $\mathrm{dACC}$ were also associated with mental rotation performance obtained during cognitive testing (normalized duration, $\rho=-0.56, p<0.01$, figure $4 c$ ).

\section{Differential role of glucose metabolism and functional connectivity in neuroplasticity}

In a simulation analysis, we disentangled the individual contributions of CMRGlu and FC to the training-induced changes in MCM described above (figure 5). This revealed that learning specific increases in MCM at resting-state were dependent on CMRGlu, but not FC for the two connections (insula and $\mathrm{dACC}$ towards Occ). Conversely, the inverse pattern was observed for the hard task condition, where MCM training effects were driven by FC, but were largely independent of CMRGlu for both connections. However, random removal of voxels up to $90 \%$ did not affect the learning-induced MCM changes. This further supports the specificity of CMRGlu and FC driving MCM training changes and indicates that effects were not dependent on the size of the target region.

\section{Structural imaging}

There were no significant learning effects regarding gray matter volume or white matter microstructure (group*time interaction, all p>0.05 FWE-corrected). 


\section{DISCUSSION}

We employed multimodal brain network analyses of simultaneous PET/MR imaging to investigate learning-induced neuroplastic changes in functional network reorganization and the underlying metabolic demands that relate to cognitive performance improvements. 4-week training of a visuo-spatial processing task resulted in adaptations of MCM from the salience network to the occipital cortex, with an increased association between glucose metabolism and BOLD-derived functional connectivity at resting-state, but decreases during the execution of the hard task condition. This divergence between resting-state and task-related MCM adaptations also explained cognitive performance after learning but was not simply driven by gray or white matter changes. Simulations further enabled a specific attribution of training effects at resting-state to CMRGlu and those during task execution to FC. Together, these findings highlight novel aspects of skill learning, where the interaction of both metabolically expensive general neuroplastic adaptations and task-specific network reorganizations is required for improvement in behavioral performance.

\section{Network-specific involvement in task execution and learning}

Similar to our previous work (Hahn et al., 2020), pronounced task-specific activations were observed for the DAN and Occ. This result complies with the employed task as Occ is involved in visual attention-drawing (Al-Aidroos et al., 2012; Bisley, 2011) and provides spatial representations of the visual field (Grill-Spector \& Malach, 2004; Somers \& Sheremata, 2013), whereas the DAN is known for its role in controlling visuo-spatial attention (Vossel et al., 2014). However, the DAN showed no relevant training effects, indicating that this network mirrors a more general involvement in visuo-spatial processing required for planning and problem solving of the current task.

In comparison, the main training effect was a pronounced alteration of the influence from the SN (insula and dACC) to Occ. As part of the $\mathrm{SN}$, the right anterior insula mediates switching between task-irrelevant networks and the activation of task-specific networks that convey externally oriented attention (Sridharan et al., 2008; Menon \& Uddin, 2010; Goulden et al., 
2014; Uddin, 2015). For instance, visual stimuli can be processed by the anterior insula through the dorsal visual pathway and the intraparietal sulcus (Menon \& Uddin, 2010; Uddin et al., 2010). The dorsal visual pathway thus represents a crucial bottom-up connection to the SN. Moreover, the anterior insula functions as an essential hub to filter relevant bottom-up stimuli and to facilitate access to working memory resources, explaining its involvement in multiple sensory and cognitive fields (Cauda et al., 2012; Kurth et al., 2010; Menon \& Uddin, 2010). Among others, anterior insula and the dACC were identified to exert activation patterns preceding task errors (Eichele et al., 2008) and were associated with performance monitoring (Ullsperger et al., 2014). Since training led to a marked improvement of task performance, a task-specific decrease of insula and dACC input to Occ during task execution might indicate a reduced error rate. The finding that changes in cognitive performance and $\mathrm{MCM}$ were mainly observed for the hard task condition is well in line with the experimental design of our Tetris ${ }^{\circledR}$ task, where only the hard level was set to require specific training. In accordance, a previous study showed that activation of the $\mathrm{SN}$ is dependent on cognitive load ((Bud) Craig, 2009; Deary et al., 2004). The accompanying behavioral data further suggest that improvements in task performance were specific to abilities of mental rotation and, to a lesser extent, visual search and working memory, but not spatial planning and problem solving. Following the assumption that mental rotation reflects a continuous transformation performed on visual representations in the human brain (Zacks, 2008), we suggest that the observed improvement is based on the representational adaptation described below.

Generally, the dACC plays an essential role in monitoring cognitive control by adjusting automatic behavioral patterns to meet specific task demands (Botvinick et al., 2001; Shenhav et al., 2013; Ullsperger et al., 2014). In more detail, dACC activation is associated with occurring conflicts between control signals (Botvinick et al., 2001; Oehrn et al., 2014; Sheth et al., 2012; Tang et al., 2016), error monitoring (Heilbronner \& Hayden, 2016; Ito et al., 2003; Shen et al., 2015) and negative feedback (Amiez et al., 2012). Inhibitory transcranial direct current stimulation of the dACC leads to decreased neuronal responses following errors and negative feedback, which subsequently results in poorer accuracy and reduced learning 
(Reinhart et al., 2015). These essential cognitive functions have led to an integrative account of dACC function to provide task representations (Heilbronner \& Hayden, 2016). Optimization of task representations through adaptive coding of task-relevant variables enables cognitive control and guidance of behavior. It was further proposed that the adjustments of dACC connections represent a flexible process, emphasizing its role in learning (Heilbronner \& Hayden, 2016).

To sum up, the observed adaptations of SN connections indicate an optimized hierarchical top-down influence onto the Occ. In this context, the anterior insula seems to play a unique role as the switching point between bottom-up saliency and top-down control, whereas the dACC provides monitoring of cognitive control and adjustment of task representations. These mechanisms emphasize a successive shift from basic task execution, instantly implemented by feedforward activation through Occ to the DAN (Hahn et al., 2020), to optimized top-down control of salient input from the $\mathrm{SN}$ to Occ after successful training, thus enabling improved cognitive performance.

\section{Cognitive skill learning through optimization of representational advancements}

We observed adaptations of MCM at both resting-state and task execution. Although these effects diverged they seem to constitute complementary adaptations of the learning process. We thereby provide a unified interpretation of skill learning as an advancement of neuronal representations of the task. While the retinal image is faithfully represented by early visual pathway areas, higher-order brain regions create edited representations of the visual field to fulfil immediate goals of attention and behavior as a function of task demands (Maunsell, 1995). Our findings thus integrate the spatio-temporal dynamics underlying bottom-up and top-down attentional control via the SN (Menon \& Uddin, 2010) into the conception of learning to reduce prediction errors between task representations of brain regions of different processing levels, based on synaptic modifications (Feldman \& Friston, 2010). 
The initial stage of the learning process is characterized by the interaction with unknown task demands and high perceptional load, thus requiring adaptation of attention due to limited processing resources (Buschman \& Kastner, 2015; Desimone \& Duncan, 1995). Attention can be understood as a Bayesian optimization of hierarchical perception to infer the precision of a probabilistic representation of the environment (Feldman \& Friston, 2010; Friston, 2005). The model is also supported by rodent studies, where projections from the ACC to the visual cortex encoded the discrepancy between predicted and actual sensory input (Fiser et al., 2016; Leinweber et al., 2017; Walsh et al., 2020; Zhang et al., 2014). The attentional optimization is implemented by recurrent loops between different hierarchical levels of cortical systems (Friston \& Kiebel, 2009; Mumford, 1992), based on synaptic gain or responsiveness of postsynaptic neurons that encode prediction errors (Friston, 2005, 2008). Within these loops socalled state and error units characterize the different representations and their inaccuracy (i.e., the prediction error), respectively (Feldman \& Friston, 2010). The prediction error itself is optimized through top-down modulatory control (Feldman \& Friston, 2010), e.g., by the SN (Menon \& Uddin, 2010). Thus, attention selects (Knudsen, 2007; Moore \& Zirnsak, 2017; Parks \& Madden, 2013) and sharpens (Buschman \& Kastner, 2015) the representations that enter working memory. Subsequently, cognitive control enables to distinguish between efficient and unsuccessful task rules, thereby implementing an advanced representation of the task (Buschman \& Kastner, 2015; Miller \& Cohen, 2001).

Training will continuously alter the task representation by incorporating relevant and discarding irrelevant information, approaching an optimal solution and finally yielding a sparse representation encoded only by a small portion of neurons (Rogerson et al., 2014). This may enable faster processing through inhibition of neurons that encode irrelevant features (GrillSpector et al., 2006). Following a sufficiently long training period, the task representation is saved within these few state units. We refer to this stored representation as 'skill engram', extending the term 'engram', first introduced in 1904 to describe memory representations (Josselyn \& Tonegawa, 2020). For the herein employed task the skill engram is equivalent to a specific pattern of functional connectivity that matches the underlying metabolic demands 
between $\mathrm{SN}$ and Occ as reflected by MCM increases at resting-state. Importantly, the stored skill engram can be retrieved instantaneously at the next task performance. Then, the optimized representations yield a small prediction error between higher- and lower-order brain regions. A minimized prediction error requires only minor cognitive control, resulting in decreased directional influence (lower MCM values) from SN to Occ during task execution. We would like to note that these representations can still be subject to adaptations and reconsolidation with further training, highlighting that engrams are dynamic in nature (Dudai, 2012; Mau et al., 2020; DeNardo et al., 2019). This strongly emphasizes that the observed effects at resting-state and during task execution complement each other by representing the stable and fluid/plastic components of memory (or skill) traces, respectively (Mau et al., 2020). The above interpretation is further supported by our observation that a high divergence of neuroplastic changes between rest and task was associated with improved task performance.

\section{Synaptic modifications, metabolic cost and network adaptations underlying skill learning}

By providing a neurobiological explanation of our findings, we acknowledge that these interpretations rely on previous work mostly obtained from preclinical studies. We propose that the dynamic process of representational advancement is molecularly implemented by synaptic tagging and capture, together referred to as long-term potentiation (Caroni et al., 2014; Herring \& Nicoll, 2016; Redondo \& Morris, 2011), whereas the skill engram's storage and retrieval might comply with synaptic (re)consolidation.

Neuronal stimulation through task performance elicits synaptic tagging, which describes shortlasting adaptations of the postsynaptic density of dendritic spines. We interpret a high number of tagging events as high prediction error, since numerous different traces are explored, which is reflected in high task-specific MCM before the learning process. Repeated tagging through continuous training of the task then induces synaptic capture, where plasticity-related products (e.g., ARC, AMPA receptor subunit GluR1) are mobilized to stabilize the spine architecture. Furthermore, the sharpening of representations might involve the stabilization process of synaptic clustering, i.e., the addition of new spines to dendritic sites that have been captured 
during earlier training sessions (Fu et al., 2012; Rogerson et al., 2014; Kastellakis et al., 2015; Frank et al., 2018). These structural adjustments enable functional synaptic potentiation by anchoring additional postsynaptic AMPA receptors (Choi et al., 2018), finally representing the consolidated skill engram. Importantly, the synaptic adaptations of the learning process beyond tagging are metabolically expensive (Plaçais et al., 2017). Particularly the insertion of AMPA receptors has been shown to double the postsynaptic energy consumption in terms of ATP (Harris et al., 2012). Since BOLD-derived FC reflects glutamate-mediated processes (Logothetis et al., 2001), we propose that the observed MCM increases at rest (i.e., the increased association between FC and CMRGlu) indicate long-term potentiation via an increased expression of glutamatergic AMPA receptors. As mentioned above, this consolidated engram obtained through training results in a low prediction error during task execution and thus also in few additional tagging events, which is reflected in a decreased task-specific MCM value after training (summarized in figure 6).

Recent metabolic simulations of synaptic adaptations supported the neurobiological process of synaptic tagging and capture as a plausible physiological mechanism to increase energy efficiency (Li \& van Rossum, 2020). It is based on a molecular optimization where a vast space of potential synaptic connections is explored to find the most appropriate representation in an energy-efficient manner (Mau et al., 2020). Computations emphasized that storage of transient memories without protein synthesis is up to ten-fold more energy efficient, compared to the immediate formation of long-term potentiation that requires a high amount of metabolic resources (Li \& van Rossum, 2020). These considerations are also in line with our own simulation experiment, indicating that energy demanding (synaptic capture) learning effects at rest were dependent on CMRGlu, while transient storage of information (synaptic tagging) elicited by task execution was driven by FC. Combining the theories above, we speculate that the simulated removal of voxels based on CMRGlu equals a perturbation of state units that represent the skill engram at resting-state. On the other hand, FC-based deletion may disrupt the ability to establish a correct prediction error during task execution as encoded by error units. Thus, the two aspects will either alter the molecular basis of neuronal task 
representations or the actual cognitive control, respectively. However, disruption of any of the two will nullify the training effect, again highlighting the complementary importance of both effects for successful learning.

\section{Limitations, outlook and conclusions}

In sum, the combined assessment of brain network dynamics and their underlying metabolic demands enabled us to describe novel complementary aspects of learning. We are aware that causal relationships between the observed findings from human brain imaging and more invasive work underpinning the prediction error model as well as the process of synaptic tagging and capture still need to be established. Although these models can be unified in our data, future work should aim to resolve this knowledge gap by directly testing the suggested associations. Another limitation of our work is that only two time points of the learning process were assessed (baseline and 4-week training). In this context, future investigations should determine the minimum training time to obtain an effective skill engram and how long it remains stable after the training is terminated. Our behavioral data suggest that efficient task execution persists at least after four weeks without further training. Finally, it needs to be determined if vulnerable development phases or adverse life events may alter the learning process. Disentangling the investigation of metabolic and functional requirements for neuroplasticity might prove beneficial to differentiate between different forms of neurodegenerative diseases and evaluate the severity of tissue damage in traumatic brain injury. Furthermore, the approach might prove valuable for assessing cognitive deficits in psychiatric disorders like depression or schizophrenia and their respective treatment. Thus, the application in patient cohorts may identify if pathological deficits in learning and memory are driven by the failure to establish the engram on a molecular basis or insufficient functional error optimization. 


\section{METHODS}

The cognitive task as well as PET/MRI data acquisition and first level analyses have been described in detail in our previous cross-sectional work (Hahn et al., 2020).

\section{Experimental design}

In this longitudinal study participants were randomly assigned to the learning or passive control group (dynamic balanced randomization stratified by age, sex and general intelligence) and underwent two PET/MRI measurements (training: $28.0 \pm 1.2$ days apart, control: $29.5 \pm 5.0$ days, figure 1). The training group practiced the cognitive task regularly between the two imaging sessions while the control group did not perform a task. After the second PET/MRI scan, also the training group stopped practicing the cognitive task, but completed a last task session on a laptop at the final visit to assess long-term skill consolidation (30.6 \pm 2.8 days after the second PET/MRI scan).

During each PET/MRI measurement subjects performed a challenging cognitive task at two predefined levels of difficulty (easy, hard). The PET/MRI acquisition lasted 100 min and has been described in detail in previous work(Hahn et al., 2020). Briefly, this included a T1weighted structural (8 $\mathrm{min}$ ) and diffusion weighted scan (12 $\mathrm{min}$ ) as well as blood oxygen level dependent (BOLD), arterial spin labeling (ASL) and simultaneous fPET imaging (supplementary figure S1). Prior to the administration of the radiotracer $\left[{ }^{18} \mathrm{~F}\right] \mathrm{FDG}, \mathrm{BOLD}$ and ASL was acquired at rest. Subjects were instructed to look at a crosshair, relax and not focus on anything in particular. Afterwards, fPET started with an initial baseline $(8.17 \mathrm{~min})$ and subsequent periods of continuous task performance $(6 \mathrm{~min}$ for two easy and two hard conditions, randomized). During the task, BOLD and ASL data were recorded in pseudorandomized order. BOLD data acquired at rest and during continuous task performance was used for the computation of metabolic connectivity mapping. All periods of task execution were followed by periods of rest $(5 \mathrm{~min})$. After fPET was completed, BOLD data were also acquired in a conventional block design (four $30 \mathrm{~s}$ blocks of easy, hard and control conditions 
each, randomized with $10 \mathrm{~s}$ baseline in-between, $8.17 \mathrm{~min}$ ) to obtain another proxy of taskspecific activation.

\section{Cognitive tasks and online training}

The cognitive task comprised an adapted version of the video game Tetris® (Hahn et al., 2020) (https://github.com/jakesgordon/javascript-tetris, MIT license), which was chosen for various reasons. First, a computerized task enables execution during the training period and also within the PET/MRI scanner (as opposed to e.g., juggling (Sampaio-Baptista et al., 2015; Scholz et al., 2009)), offering the key possibility to assess learning effects at rest and during task performance. Also, the individual task performance can be easily monitored to follow the training success of the participants. Furthermore, the task is well suited to maintain high levels of attention during prolonged and continuous task performance, which in turn provides an optimal setting for fPET imaging (Rischka et al., 2018). Finally, the computerized task enables programming of different levels of task difficulty. Thereby, we intentionally set the easy level to be manageable for a novice, whereas the hard level requires specific training. We therefore expected the most pronounced effects regarding behavioral data and neuroplasticity for the hard task condition. In this task bricks of different shape drop from the top of the screen. By rotation and alignment of the bricks, the aim is to build complete horizontal lines which disappear and increase the score. Bricks were moved by operating four buttons with the right hand only (index finger: move brick left, middle: rotation, ring: down, small: right; same fixed assignment for in-scanner and online version).

For the PET/MRI measurements, the two levels of difficulty differed regarding the speed of the falling bricks (easy/hard: 1/3 lines per sec) and the number of incomplete lines that were already built at the bottom (easy/hard: $2 / 6$ lines out of 20). An additional control condition was employed for the BOLD block design, where bricks were navigated through a chimney and disappeared at the bottom. Participants familiarized themselves with the task and control buttons by a $30 \mathrm{~s}$ training of each task condition right before the scan started. For the online learning phase between the two PET/MRI scans participants were instructed to train on a 
regular basis (45-60 min per session, at least 20 sessions within 4 weeks). An individual username and password were provided to access the web-based training platform in the web browser Chrome. Subjects were able to select any combination of brick speed and prebuilt lines freely. However, the explicit aim of the 4-week training period was to be able to manage the hard task condition as carried out in the PET/MRI session. The implemented scoring was identical for the PET/MRI measurements and online training, following the original scheme of Tetris $®$. The score for each complete line was $k^{*}(n+1)$, with $n$ being the speed level of the falling bricks and k representing the score for one (40), two (100), three (300) or four (1200) lines removed simultaneously.

At each PET/MRI examination, participants also completed a cognitive testing session outside the scanner to relate improvements in task performance to specific cognitive domains of mental rotation, visual search and working memory, as well as spatial planning and problem solving. For the mental rotation task, subjects viewed pairs of 3D objects (similar to Tetris bricks) and indicated if the objects are congruent after mental rotation or not (Shepard \& Metzler, 1971) (images obtained from http://www.tarrlab.org/ under CC BY-NC-SA 3.0). The paradigm comprised 20 stimuli pairs of $40^{\circ}, 80^{\circ}, 120^{\circ}$, and $160^{\circ}$ rotation (5 each) and $50 \%$ congruent pairs (random presentation order). Abilities of visual search and working memory were tested with the delayed match to sample task (Daniel et al., 2016). After the presentation of an abstract pattern (10x10 square of black and white tiles) for $1 \mathrm{~s}$ and a delay of another second, subjects were required to choose the matching pattern from a set of $2,4,6$, or 8 similar patterns (3 each, 12 stimuli sets in total, random presentation order). Spatial planning and problem solving abilities were assessed with the Tower of London task (Shallice et al., 1982; Unterrainer et al., 2004). Within the task, starting from an initial configuration of three colored discs on three pegs with different height, subjects are required to move the discs to reach a specific target configuration. The paradigm included 3-8 moves ( 2 each) and 12 stimulus sets in total (random presentation order). 


\section{Participants}

For this study, 53 healthy subjects were initially recruited and data from 41 healthy subjects were included in the analysis (all right-handed). Reasons for study drop out were voluntary discontinuation $(n=6)$, omission to acquire fPET due to issues with arterial cannulation or radiotracer synthesis $(n=3)$, failure of arterial blood sampling $(n=2)$ and excessive head motion during the BOLD acquisition ( $n=1)$. Among the 41 subjects, 21 were assigned to the training group (mean age $\pm s d=23.0 \pm 3.6$ years, 11 women) and 20 to the control group $(23.1 \pm 3.1$ years, 10 women). For two subjects of the training group, no ASL data were available due to technical issues. As no longitudinal MCM studies are available, the sample size was based on previous cross-sectional work using this technique (Hahn et al., 2020; Riedl et al., 2016). Parts of this sample were already included in previous studies for the assessment of cross-sectional data (Hahn et al., 2020) and test-retest reliability (Rischka et al., 2021). At the screening visit, general health of all subjects was ensured through a routine medical examination performed by an experienced psychiatrist, including blood tests, electrocardiography, neurological testing (comprising examinations of mental status, cranial nerves, motor system, deep tendon reflexes, sensation, cerebellum) and the structural clinical interview for DSM-IV. All subjects also underwent a shortened version of the Raven standard progressive matrices test at the screening visit as an index of general intelligence (training group: $113.3 \pm 9.5$, control group: $115.1 \pm 9.0$; two parameter logistic model (Van der Elst et al., 2013)). Urine pregnancy tests were carried out for female participants at the screening visit and before each PET/MRI measurement. Exclusion criteria were current and previous somatic, neurological or psychiatric disorders (12 months), substance abuse or psychopharmacological medication (6 months), current pregnancy or breast feeding, previous study-related radiation exposure (10 years), body weight of more than $100 \mathrm{~kg}$ for reasons of radiation protection, MRI contraindications and previous experience with the video game Tetris ${ }^{\circledR}$ or similar games ( 3 years). After a detailed explanation of the study protocol, all participants provided written informed consent, and they were insured and, after study completion, reimbursed for participation. The study was approved by the ethics committee of the Medical University of Vienna (ethics number: 
1479/2015) and procedures were carried out in accordance with the Declaration of Helsinki.

The study was pre-registered at ClinicalTrials.gov (NCT03485066).

\section{PETMRI data acquisition}

Participants had to fast for at least 4 hours before the start of the PET/MRI scan (except for unsweetened water). The radiotracer 2-[ $\left.{ }^{18} \mathrm{~F}\right]$ fluoro-2-deoxy-D-glucose $\left[{ }^{18} \mathrm{~F}\right] \mathrm{FDG}$ was administered in a bolus $(510 \mathrm{kBq} / \mathrm{kg} / \mathrm{frame}$ for $1 \mathrm{~min})$ plus infusion protocol $(40 \mathrm{kBq} / \mathrm{kg} / \mathrm{frame}$ for $51 \mathrm{~min}$ ) with a perfusion pump (Syramed $\mu$ SP6000, Arcomed, Regensdorf, Switzerland) which was kept in an MRI-shield (UniQUE, Arcomed) (Rischka et al., 2018).

MRI recordings included a structural T1-weighted acquisition (MPRAGE sequence, TE/TR = $4.21 / 2200 \mathrm{~ms}, \mathrm{TI}=900 \mathrm{~ms}$, flip angle $=9^{\circ}$, matrix size $=240 \times 256,160$ slices, voxel size $=1$ $\mathrm{x} 1 \mathrm{x} 1 \mathrm{~mm}+0.1 \mathrm{~mm}$ gap, $7.72 \mathrm{~min})$ and diffusion weighted imaging (EPI sequence, TE/TR = $86 / 8800 \mathrm{~ms}, 64$ diffusion directions with $\mathrm{b}$-value $=1000 \mathrm{~s} / \mathrm{mm}^{2}, 6$ b0-images, matrix size $=104$ x 104, 70 slices, voxel size $=2 \times 2 \times 2 \mathrm{~mm}, 11.73 \mathrm{~min})$.

Simultaneously with fPET, functional MRI was obtained using ASL (2D pseudo-continuous ASL sequence, $\mathrm{TE} / \mathrm{TR}=12 / 4060 \mathrm{~ms}$, post label delay $=1800 \mathrm{~ms}$, flip angle $=90^{\circ}$, matrix size $=64 \times 64,20$ slices, voxel size $=3.44 \times 3.44 \times 5$ mm +1 mm gap, $3 \times 6$ min (Kilroy et al., 2014)) and BOLD imaging (EPI sequence, TE $/$ TR $=30 / 2000 \mathrm{~ms}$, flip angle $=90^{\circ}$, matrix size $=80 \mathrm{x}$ 80,34 slices, voxel size $=2.5 \times 2.5 \times 2.5 \mathrm{~mm}+0.825 \mathrm{~mm}$ gap, $3 \times 6 \mathrm{~min}$ for functional connectivity and 8.17 min for neuronal activation in the block design).

\section{Blood sampling}

Blood glucose levels were assessed right before the PET/MRI scan (Gluplasma, triplicate). Manual arterial blood samples were taken at 3, 4, 5, 14, 25, 36 and 47 min after starting the radiotracer administration, providing a sufficiently sampled input function for the used bolus plus infusion protocol (Rischka et al., 2018). For all samples, whole blood activity and following centrifugation also plasma activity were measured in a gamma-counter (Wizard ${ }^{2}$, Perkin 
bioRxiv preprint doi: https://doi.org/10.1101/2021.11.12.468351; this version posted November 12, 2021. The copyright holder for this preprint (which was not certified by peer review) is the author/funder, who has granted bioRxiv a license to display the preprint in perpetuity. It is made available under aCC-BY-NC-ND 4.0 International license.

Elmer). Whole blood activities were linearly interpolated to match PET frames and multiplied with the average plasma-to-whole-blood ratio, yielding the arterial input function.

\section{Cerebral metabolic rate of glucose metabolism (CMRGlu)}

The reconstruction and processing of fPET data were carried out as described previously (Rischka et al., 2018). PET list mode data were corrected for attenuation with an established database approach (Burgos et al., 2014) and reconstructed to frames of $30 \mathrm{~s}$ (matrix size $=$ 344 x 344, 127 slices). Preprocessing was done in SPM12 (https://www.fil.ion.ucl.ac.uk/spm/) and included motion correction (quality $=1$, register to mean), spatial normalization to $\mathrm{MNI}$ space via the T1-weighted structural MRI and spatial smoothing with an $8 \mathrm{~mm}$ Gaussian kernel. Masking was applied to include only gray matter voxels and a low pass filter was employed with the cutoff frequency being 3 min (i.e., half the task duration). The general linear model was used to separate baseline from task-specific effects. The four regressors described the baseline, both task conditions (easy and hard, linear ramp function with slope $=1 \mathrm{kBq} / \mathrm{frame}$ ) and head motion (first principal component of all six motion regressors). The baseline regressor was computed as average time course of all gray matter voxels, excluding those activated during the hard task of the individual BOLD block design $(p<0.05$ FWE-corrected at voxel level). This multimodal approach has been shown to provide the best model fit (Rischka et al., 2018). Furthermore, including the BOLD changes in the baseline definition does not affect fPET task effects (Rischka et al., 2018) or their test-retest reliability (Rischka et al., 2021). For absolute quantification of glucose metabolism, the Patlak plot was applied to calculate the influx constant, $\mathrm{K}_{\mathrm{i}}$. This was subsequently converted to CMRGlu by

$$
\text { CMRGlu }=K_{i} * \text { Gluplasma } / \text { LC * } 100
$$

with the lumped constant (LC) set to 0.89 . This procedure yields separate maps of CMRGlu at baseline and for each task condition (easy, hard), which were then used as an index of neuronal activation and for computation of metabolic connectivity mapping (see below). The approach has been shown to yield excellent test-retest reliability for CMRGlu at rest and fairto-good reliability during task performance (Rischka et al., 2021). 


\section{Cerebral blood flow (CBF)}

ASL data were processed according to standard procedures (Wang et al., 2005). Voxels with a signal intensity below 0.8 times the mean value were set to zero to remove spurious effects, followed by motion correction in SPM12 (quality $=1$, register to mean). The equilibrium magnetization of the brain $M_{0}$ was calculated as average of all non-labeled images. $A$ brain mask was computed from the $M_{0}$ image with the brain extraction tool (FSL, https://fsl.fmrib.ox.ac.uk/fsl/fslwiki/). All data were masked accordingly, spatially normalized to MNI space via the T1-weighted MRI (as for the fPET data) and spatially smoothed with a $8 \mathrm{~mm}$ Gaussian kernel. CBF was then calculated by

$$
C B F=\frac{\lambda \Delta M R_{1 a}}{2 \alpha M_{0}\left\{\exp \left(-\omega R_{1 a}\right)-\exp \left[-(\tau+\omega) R_{1 a}\right]\right\}}
$$

In this equation $\lambda=0.9 \mathrm{ml} / \mathrm{g}$ represents the blood-tissue water partition coefficient, $\Delta \mathrm{M}$ the pairwise difference between labeled and non-labeled images, $R_{1 a}=0.67 \mathrm{~s}^{-1}$ the longitudinal relaxation rate of blood, $\alpha=0.8$ the tagging efficiency, $\omega$ the post-labeling delay adapted for slice timing effects ( $=1800$ at slice 1$)$ and $\tau=1508 \mathrm{~ms}$ the duration of the labeling pulse. CBF was then averaged across time series separately for each condition (rest, easy, hard). As the maps obtained during task performance represent the sum of resting and task effects, the CBF at rest was subtracted to finally obtain the sole task-specific changes in CBF as a further proxy of neuronal activation.

\section{Blood oxygen level dependent (BOLD) signal changes}

BOLD data of the block design were processed with SPM12 as described previously (Rischka et al., 2018). Data were corrected for slice timing effects (reference $=$ middle slice) and head motion (quality $=1$, register to mean). Similar to fPET and ASL, normalization to MNI space was carried out via the T1-weighted MRI, followed by spatial smoothing with an $8 \mathrm{~mm}$ Gaussian kernel. Task-specific changes were estimated with the general linear model, which included one regressor for each task condition (easy, hard, control) and nuisance regressors for motion, 
white matter and cerebrospinal fluid. The contrasts of interest used for the subsequent analyses were easy vs. control and hard vs. control.

\section{Overlap in task-specific neuronal activation}

The three different indices of task-specific metabolic demands (CMRGlu, CBF, BOLD) were combined to obtain a robust estimate of regions involved in task processing. For each imaging modality, separate one sample t-tests were performed for the hard task condition of the first PET/MRI measurement across the entire sample. As ASL data were missing for two subjects, the sample used for this overlap analysis was $n=39$. The resulting statistical maps were thresholded ( $p<0.05$ FWE corrected voxel level), binarized and combined in a conjunction analysis by computing the intersection across all three imaging modalities (supplementary figure S2). The brain regions identified in this analysis were used for the subsequent MCM analysis, where homologous regions in left and right hemispheres were combined. The spatial overlap between the different imaging modalities was assessed by the Dice coefficient applied to the thresholded and binarized maps.

\section{Metabolic connectivity mapping (MCM)}

MCM is a multimodal framework that investigates the association of regional patterns between glucose metabolism and BOLD-derived functional connectivity (FC) (Riedl et al., 2016). Considering that the majority of energy demands emerge post-synaptically (Attwell \& Laughlin, 2001; Harris et al., 2012; Mergenthaler et al., 2013), the incorporation of CMRGlu identifies the target region and thus the $\mathrm{MCM}$ framework allows to assign directionality to a specific connection. In short, it is assumed that the seed region exerts an influence on the target region, yielding a particular voxelwise FC pattern. If this influence is indeed causal, it will result in a corresponding CMRGlu pattern due to the coupling between the BOLD signal and metabolism (Attwell et al., 2010; Mishra et al., 2016), which is reflected in a non-zero MCM value given by the spatial correlation between patterns of CMRGlu and FC. For a detailed description of the 
underlying neurobiological effects of this approach and the analysis details the reader is referred to previous work (Hahn et al., 2020; Riedl et al., 2016).

FC was computed at rest and for the two task conditions (easy, hard) from continuously acquired BOLD data using the preprocessing pipeline employed for the BOLD block design described above. After spatial smoothing, motion scrubbing was carried out to remove spurious connectivity induced by head motion (Power et al., 2015). Rotational motion parameters were converted to $\mathrm{mm}$ (displacement on a $50 \mathrm{~mm}$ sphere) and summed with the translational parameters. Frames with a displacement $>0.5 \mathrm{~mm}$ (plus one frame back and two forward) were discarded resulting in an average removal of $4.3 \pm 4.7 \%$ of frames. There was no significant difference in head motion between groups, PET/MRI measurements and conditions ( $p>0.3$ for all interactions and post-hoc tests). Subsequently, further confounding signals were removed by linear regression (motion parameters, white matter, cerebrospinal fluid) and a bandpass filter $(0.01<f 0.15 \mathrm{~Hz}$, enabling comparison of connectivity at rest and task performance (Sun et al., 2004)).

In this work, we extended the MCM framework from a region-of-interest to the whole-brain level, thereby avoiding a bias inherent to the a priori selection of brain regions. First, a specific target region $\mathrm{B}$ was chosen, herein defined as the overlap of neuronal activation across imaging modalities (see results and supplementary figure S2). Next, the BOLD signal of any single voxel $A_{i}$ in the brain was (temporally) correlated with the time course of all voxels in the target region $\mathrm{B}$ and z-transformed, yielding a voxel-wise pattern of $\mathrm{FC}$ in $\mathrm{B}$. This FC pattern of $\mathrm{B}$ was then (spatially) correlated with the corresponding CMRGlu pattern of $\mathrm{B}$. The resulting MCM value was again z-transformed and assigned to the voxel $A_{i}$. Repeating the computation for every voxel $A_{i}$ in the brain gives a whole-brain map of $M C M$, where each voxel $A_{i}$ represents the directional connectivity to the target region $\mathrm{B}$.

Previous studies have demonstrated that MCM is not affected by the size of the target region (Riedl et al., 2016), spatial smoothing or the preprocessing order of FC data (Hahn et al., 2020). Furthermore, directionality inferred from MCM has been validated by dynamic causal modeling (Hahn et al., 2020). 


\section{Gray matter volume}

T1-weighted structural images were segmented and spatially normalized to MNI-space using the longitudinal pipeline implemented in the CAT12 toolbox for SPM12 with default parameters to detect learning-induced changes. Gray matter volume was calculated by multiplication of the corresponding segment with the Jacobian determinants, adjusting for nonlinear deformation effects. The resulting images were spatially smoothed with an $8 \mathrm{~mm}$ Gaussian kernel.

\section{White matter microstructure}

Diffusion weighted images were processed with FSL as described previously (Gryglewski et al., 2020). This included removal of nonbrain tissue with the brain extraction tool as well as correction for eddy currents, head movement and distortions with outlier replacement. For estimation of the diffusion tensors, the rotated b-vectors as obtained during the previous step were used, resulting in images of axial, radial and diffusivity as well as fractional anisotropy (FA). Spatial normalization to MNI space was carried out with tract based spatial statistics (Smith et al., 2006) by creating a white matter skeleton from the mean FA image and subsequent mapping of individual FA images. The obtained transformations were applied to the three diffusivity metrics.

\section{Statistical analysis}

All statistical tests were two-sided and the reported $p$-values were corrected for multiple comparisons. For imaging data, this was realized with family wise error correction (MCM and gray matter volume: $p<0.05$ FWE cluster level, following $p<0.001$ uncorrected voxel level in SPM12; white matter microstructure: $p<0.05$ FWE-corrected with threshold free cluster enhancement, 500 random permutations in FSL). For behavioral data, head motion and all post-hoc tests, the corrections were done with the Bonferroni-Holm procedure (number of performed tests for each analysis given below in brackets). 
Behavioral data acquired during the execution of Tetris ${ }^{\circledR}$ was defined as the score per minute, allowing a comparison between task performance during the PET/MRI scans and the online training. For the mental rotation task, the overall duration to solve all image pairs (low = good performance) divided by the number of correct answers (high = good performance) was used as a summary measure. For the Tower of London and match-to-sample tasks, the processing time and the number of correct answers were used as the outcome, respectively. Behavioral data were analyzed with a repeated measures ANOVA in Matlab with factors group (control, training), time (PET/MRI scan 1 and 2) and condition (easy, hard). Training-induced changes were assessed by the interaction effect group * time * condition (one interaction). In the posthoc analysis the interaction group * time was tested for each condition separately (two conditions), followed by paired t-tests of the time effect within each group (two groups). Independent-samples t-tests were used to assess differences in initial task performance between the training and control group (two conditions). For the training group, additional paired t-tests were conducted to test for differences in Tetris $®$ score between the second PET/MRI scan and the final visit (two conditions).

Imaging data were evaluated in a similar manner, testing for training-induced changes in MCM. First, a repeated measures ANOVA was implemented in SPM12 with factors group (control, training), time (PET/MRI scan 1 and 2) and condition (rest, easy, hard). As SPM allows only two factors (in addition to the subject factor), the factor time was implicitly included by entering the difference maps between PET/MRI scan 2 vs 1 into the model. Based on our experimental design and the subsequent expectation that the hard task level will show the strongest neuroplastic changes, we tested for a conventional interaction effect. However, considering previous similarity of MCM effects between the easy and the hard task conditions (Hahn et al., 2020) a second contrast of interest was chosen as rest vs. (easy + hard). For the subsequent post-hoc analysis regional MCM values were extracted from the significant clusters and analyzed in Matlab. Here, the interaction group * time was tested for each condition separately (three conditions), followed by paired t-tests of the time effect within each group (two groups). 
MCM values at measurement 2 (i.e., after training) were correlated with the corresponding behavioral data. Based on our findings (see results), the divergence between MCM increases at rest and decreases during the task can be summarized by their difference. Normalization by the mean value further takes the absolute value into account while avoiding mathematical coupling (Beggs et al., 2020). The MCM difference was also correlated with the overall learning performance, quantified by the normalized area under the curve of scoring obtained during the entire 4-week training period. Spearman's rho was calculated to account for one outlying value. Structural changes in gray matter volume and white matter microstructure were calculated by repeated measures ANOVA in SPM12 and FSL, respectively. The analysis included the factors group (control, training) and time (PET/MRI scan 1 and 2), testing for their interaction.

Differences in head motion (i.e., framewise displacement) during FC acquisition were assessed by repeated measures ANOVA in Matlab with factors group (control, training), time (PET/MRI scan 1 and 2) and condition (rest, easy, hard). Again, we tested the interaction effect group * time * condition (one interaction) and post-hoc interactions group * time for each condition separately (three conditions).

\section{Simulations}

Simulations were carried out to identify whether the training-specific changes in MCM were driven by CMRGlu or FC. As MCM represents the spatial correlation between voxel-wise patterns of CMRGlu and FC, voxels in the target region were removed in a step-wise manner and the training effects (i.e., F-value of group*time interactions) were re-calculated. Thus, a decrease in the training effect is expected if the observed changes in MCM are indeed driven by one of the parameters.

First, voxels of the target region were removed randomly and the resulting MCM z-scores were averaged for 500 random selections of voxels. Second, voxels were progressively removed based on the amplitude values of the two imaging parameters. More specifically, voxels were removed either on the basis of the underlying CMRGlu or FC values, starting with those voxels 
bioRxiv preprint doi: https://doi org/10.1101/2021.11.12.468351; this version posted November 12, 2021. The copyright holder for this preprint (which was not certified by peer review) is the author/funder, who has granted bioRxiv a license to display the preprint in perpetuity. It is made available under aCC-BY-NC-ND 4.0 International license.

that contained the lowest values. For both the random and specific approaches, an increasing amount of voxels were removed in steps of $10 \%$ up to $90 \%$. 


\section{ACKNOWLEDGEMENTS}

The authors are particularly grateful to Prof. Danny JJ Wang, Stevens Neuroimaging and Informatics Institute, University of South California, and the Regents of the University of California, for providing the pCASL sequence. Stimulus images for the mental rotation task are courtesy of Michael J. Tarr, Carnegie Mellon University, http://www.tarrlab.org/. We thank the graduated team members and the diploma students of the Neuroimaging Lab (NIL, head: R. Lanzenberger) as well as the clinical colleagues from the Department of Psychiatry and Psychotherapy for clinical and/or administrative support. In detail, we would like to thank S. Kasper, K. Papageorgiou, P. Michenthaler, T. Vanicek, A. Basaran, M. Hienert, L. Silberbauer, J. Unterholzner and G. Gryglewski medical support, M. B. Reed for analysis support, V. Ritter, K. Einenkel and E. Sittenberger for subject recruitment and A. Jelicic for partly implementation of the task. We are further grateful to J. Raitanen, J. Völkle and A. Pomberger for radioligand synthesis.

This research was funded in whole, or in part, by the Austrian Science Fund (FWF) KLI 610, PI: A. Hahn. For the purpose of open access, the author has applied a CC BY public copyright license to any Author Accepted Manuscript version arising from this submission. S. Klug is supported by the MDPhD Excellence Program of the Medical University of Vienna. L. Rischka and M. Klöbl were recipients of a DOC Fellowship of the Austrian Academy of Sciences at the Department of Psychiatry and Psychotherapy, Medical University of Vienna. The scientific project was performed with the support of the Medical Imaging Cluster of the Medical University of Vienna.

\section{AUTHOR CONTRIBUTIONS}

Study design: A.H., R.L., M.H.

Data acquisition: L.R., A.H., S.K., G.M.G., V.P., W.W., M.K.

Data analysis: A.H., L.R., M.K.

Manuscript preparation: S.K., G.M.G., A.H. 
All authors discussed the implications of the findings and approved the final version of the manuscript.

\section{CONFLICT Of INTEREST}

R. Lanzenberger received travel grants and/or conference speaker honoraria within the last three years from Bruker BioSpin MR and Heel, and has served as a consultant for Ono Pharmaceutical. He received investigator-initiated research funding from Siemens Healthcare regarding clinical research using PET/MRI. He is a shareholder of the start-up company BM Health $\mathrm{GmbH}$ since 2019. M. Hacker received consulting fees and/or honoraria from Bayer Healthcare BMS, Eli Lilly, EZAG, GE Healthcare, Ipsen, ITM, Janssen, Roche, and Siemens Healthineers. W. Wadsak declares to having received speaker honoraria from the GE Healthcare and research grants from Ipsen Pharma, Eckert-Ziegler AG, Scintomics, and ITG; and working as a part time employee of CBmed Ltd. (Center for Biomarker Research in Medicine, Graz, Austria). All other authors report no conflict of interest in relation to this study.

\section{DATA AVAILABILITY}

Raw data will not be publicly available due to reasons of data protection. Processed data can be obtained from the corresponding author with a data-sharing agreement. 


\section{REFERENCES}

Al-Aidroos, N., Said, C. P., \& Turk-Browne, N. B. (2012). Top-down attention switches coupling between low-level and high-level areas of human visual cortex. Proceedings of the National Academy of Sciences, 109(36), 14675-14680. https://doi.org/10.1073/pnas.1202095109

Amiez, C., Sallet, J., Procyk, E., \& Petrides, M. (2012). Modulation of feedback related activity in the rostral anterior cingulate cortex during trial and error exploration. Neurolmage, 63(3), 1078-1090. https://doi.org/10.1016/j.neuroimage.2012.06.023

Attwell, D., Buchan, A. M., Charpak, S., Lauritzen, M., MacVicar, B. A., \& Newman, E. A. (2010). Glial and neuronal control of brain blood flow. Nature, 468(7321), 232-243. https://doi.org/10.1038/nature09613

Attwell, D., \& Laughlin, S. B. (2001). An Energy Budget for Signaling in the Grey Matter of the Brain. Journal of Cerebral Blood Flow \& Metabolism, 21(10), 1133-1145. https://doi.org/10.1097/00004647-200110000-00001

Bassett, D. S., \& Mattar, M. G. (2017). A Network Neuroscience of Human Learning: Potential to Inform Quantitative Theories of Brain and Behavior. Trends in Cognitive Sciences, 21(4), 250-264. https://doi.org/10.1016/j.tics.2017.01.010

Bassett, D. S., Wymbs, N. F., Porter, M. A., Mucha, P. J., Carlson, J. M., \& Grafton, S. T. (2011). Dynamic reconfiguration of human brain networks during learning. Proceedings of the National Academy of Sciences, 108(18), 7641-7646. https://doi.org/10.1073/pnas. 1018985108

Beggs, L., Briscoe, R., Griffiths, C., Ellison, G. T. H., \& Gilthorpe, M. S. (2020). Intervention differential effects and regression to the mean in studies where sample selection is based on the initial value of the outcome variable: An evaluation of methods illustrated in weight-management studies. Biostatistics \& Epidemiology, 4(1), 172-188. https://doi.org/10.1080/24709360.2020.1719690

Bisley, J. W. (2011). The neural basis of visual attention. The Journal of Physiology, 589(Pt 1), 49-57. https://doi.org/10.1113/jphysiol.2010.192666

Botvinick, M. M., Braver, T. S., Barch, D. M., Carter, C. S., \& Cohen, J. D. (2001). Conflict monitoring and cognitive control. Psychological Review, 108(3), 624-652. https://doi.org/10.1037/0033-295x.108.3.624

(Bud) Craig, A. D. (2009). How do you feel - now? The anterior insula and human awareness. Nature Reviews Neuroscience, 10(1), 59-70. https://doi.org/10.1038/nrn2555

Burgos, N., Cardoso, M. J., Thielemans, K., Modat, M., Pedemonte, S., Dickson, J., Barnes, A., Ahmed, R., Mahoney, C. J., Schott, J. M., Duncan, J. S., Atkinson, D., Arridge, S. R., Hutton, B. F., \& Ourselin, S. (2014). Attenuation Correction Synthesis for Hybrid PET-MR Scanners: Application to Brain Studies. IEEE Transactions on Medical Imaging, 33(12), 2332-2341. https://doi.org/10.1109/TMI.2014.2340135

Buschman, T. J., \& Kastner, S. (2015). From Behavior to Neural Dynamics: An Integrated Theory of Attention. Neuron, 88(1), 127-144. https://doi.org/10.1016/j.neuron.2015.09.017

Caroni, P., Chowdhury, A., \& Lahr, M. (2014). Synapse rearrangements upon learning: From divergent-sparse connectivity to dedicated sub-circuits. Trends in Neurosciences, 37(10), 604-614. https://doi.org/10.1016/j.tins.2014.08.011

Cauda, F., Costa, T., Torta, D. M. E., Sacco, K., D’Agata, F., Duca, S., Geminiani, G., Fox, P. T., \& Vercelli, A. (2012). Meta-analytic clustering of the insular cortex: Characterizing the meta-analytic connectivity of the insula when involved in active tasks. Neurolmage, 62(1), 343-355. https://doi.org/10.1016/j.neuroimage.2012.04.012 
Choi, J.-H., Sim, S.-E., Kim, J., Choi, D. I., Oh, J., Ye, S., Lee, J., Kim, T., Ko, H.-G., Lim, C.S., \& Kaang, B.-K. (2018). Interregional synaptic maps among engram cells underlie memory formation. Science, 360(6387), 430-435. https://doi.org/10.1126/science.aas9204

Cole, M. W., Bassett, D. S., Power, J. D., Braver, T. S., \& Petersen, S. E. (2014). Intrinsic and Task-Evoked Network Architectures of the Human Brain. Neuron, 83(1), 238-251. https://doi.org/10.1016/j.neuron.2014.05.014

Daniel, T. A., Katz, J. S., \& Robinson, J. L. (2016). Delayed match-to-sample in working memory: A BrainMap meta-analysis. Biological Psychology, 120, 10-20. https://doi.org/10.1016/j.biopsycho.2016.07.015

Deary, I. J., Simonotto, E., Meyer, M., Marshall, A., Marshall, I., Goddard, N., \& Wardlaw, J. M. (2004). The functional anatomy of inspection time: An event-related fMRI study. Neurolmage, 22(4), 1466-1479. https://doi.org/10.1016/j.neuroimage.2004.03.047

DeNardo, L. A., Liu, C. D., Allen, W. E., Adams, E. L., Friedmann, D., Fu, L., Guenthner, C. J., Tessier-Lavigne, M., \& Luo, L. (2019). Temporal evolution of cortical ensembles promoting remote memory retrieval. Nature Neuroscience, 22(3), 460-469. https://doi.org/10.1038/s41593-018-0318-7

Desimone, R., \& Duncan, J. (1995). Neural Mechanisms of Selective Visual Attention. Annual Review of Neuroscience, 18(1), 193-222. https://doi.org/10.1146/annurev.ne.18.030195.001205

Dudai, Y. (2012). The Restless Engram: Consolidations Never End. Annual Review of Neuroscience, 35(1), 227-247. https://doi.org/10.1146/annurev-neuro-062111-150500

Eichele, T., Debener, S., Calhoun, V. D., Specht, K., Engel, A. K., Hugdahl, K., Cramon, D. Y. von, \& Ullsperger, M. (2008). Prediction of human errors by maladaptive changes in event-related brain networks. Proceedings of the National Academy of Sciences, 105(16), 6173-6178. https://doi.org/10.1073/pnas.0708965105

Feldman, H., \& Friston, K. (2010). Attention, Uncertainty, and Free-Energy. Frontiers in Human Neuroscience, 4. https://doi.org/10.3389/fnhum.2010.00215

Fiser, A., Mahringer, D., Oyibo, H. K., Petersen, A. V., Leinweber, M., \& Keller, G. B. (2016). Experience-dependent spatial expectations in mouse visual cortex. Nature Neuroscience, 19(12), 1658-1664. https://doi.org/10.1038/nn.4385

Frank, A. C., Huang, S., Zhou, M., Gdalyahu, A., Kastellakis, G., Silva, T. K., Lu, E., Wen, X., Poirazi, P., Trachtenberg, J. T., \& Silva, A. J. (2018). Hotspots of dendritic spine turnover facilitate clustered spine addition and learning and memory. Nature Communications, 9(1), 422. https://doi.org/10.1038/s41467-017-02751-2

Friston, K. (2005). A theory of cortical responses. Philosophical Transactions of the Royal Society B: Biological Sciences, 360(1456), 815-836. https://doi.org/10.1098/rstb.2005.1622

Friston, K. (2008). Hierarchical Models in the Brain. PLOS Computational Biology, 4(11), e1000211. https://doi.org/10.1371/journal.pcbi.1000211

Friston, K., \& Kiebel, S. (2009). Predictive coding under the free-energy principle. Philosophical Transactions of the Royal Society B: Biological Sciences, 364(1521), 1211-1221. https://doi.org/10.1098/rstb.2008.0300

Fu, M., Yu, X., Lu, J., \& Zuo, Y. (2012). Repetitive motor learning induces coordinated formation of clustered dendritic spines in vivo. Nature, 483(7387), 92-95. https://doi.org/10.1038/nature10844

Goulden, N., Khusnulina, A., Davis, N. J., Bracewell, R. M., Bokde, A. L., McNulty, J. P., \& Mullins, P. G. (2014). The salience network is responsible for switching between the 
default mode network and the central executive network: Replication from DCM. Neurolmage, 99, 180-190. https://doi.org/10.1016/j.neuroimage.2014.05.052

Grill-Spector, K., Henson, R., \& Martin, A. (2006). Repetition and the brain: Neural models of stimulus-specific effects. Trends in Cognitive Sciences, 10(1), 14-23. https://doi.org/10.1016/j.tics.2005.11.006

Grill-Spector, K., \& Malach, R. (2004). The Human Visual Cortex. Annual Review of Neuroscience, $27(1)$, 649-677. https://doi.org/10.1146/annurev.neuro.27.070203.144220

Gryglewski, G., Seiger, R., Baldinger-Melich, P., Unterholzner, J., Spurny, B., Vanicek, T., Hahn, A., Kasper, S., Frey, R., \& Lanzenberger, R. (2020). Changes in White Matter Microstructure After Electroconvulsive Therapy for Treatment-Resistant Depression. International Journal of Neuropsychopharmacology, 23(1), 20-25. https://doi.org/10.1093/ijnp/pyz059

Hahn, A., Breakspear, M., Rischka, L., Wadsak, W., Godbersen, G. M., Pichler, V., Michenthaler, P., Vanicek, T., Hacker, M., Kasper, S., Lanzenberger, R., \& Cocchi, L. (2020). Reconfiguration of functional brain networks and metabolic cost converge during task performance. ELife, 9, e52443. https://doi.org/10.7554/eLife.52443

Hahn, A., Gryglewski, G., Nics, L., Hienert, M., Rischka, L., Vraka, C., Sigurdardottir, H., Vanicek, T., James, G. M., Seiger, R., Kautzky, A., Silberbauer, L., Wadsak, W., Mitterhauser, M., Hacker, M., Kasper, S., \& Lanzenberger, R. (2016). Quantification of Task-Specific Glucose Metabolism with Constant Infusion of 18F-FDG. Journal of Nuclear Medicine, 57(12), 1933-1940. https://doi.org/10.2967/jnumed.116.176156

Haier, R. J., Siegel, B. V., MacLachlan, A., Soderling, E., Lottenberg, S., \& Buchsbaum, M. S. (1992). Regional glucose metabolic changes after learning a complex visuospatial/motor task: A positron emission tomographic study. Brain Research, 570(1-2), 134-143.

Harris, J. J., Jolivet, R., \& Attwell, D. (2012). Synaptic Energy Use and Supply. Neuron, 75(5), 762-777. https://doi.org/10.1016/j.neuron.2012.08.019

Hearne, L. J., Cocchi, L., Zalesky, A., \& Mattingley, J. B. (2017). Reconfiguration of Brain Network Architectures between Resting-State and Complexity-Dependent Cognitive Reasoning. The Journal of Neuroscience, 37(35), 8399-8411. https://doi.org/10.1523/JNEUROSCI.0485-17.2017

Heilbronner, S. R., \& Hayden, B. Y. (2016). Dorsal Anterior Cingulate Cortex: A Bottom-Up View. Annual Review of Neuroscience, 39, 149-170. https://doi.org/10.1146/annurevneuro-070815-013952

Herring, B. E., \& Nicoll, R. A. (2016). Long-Term Potentiation: From CaMKII to AMPA Receptor Trafficking. Annual Review of Physiology, 78(1), 351-365. https://doi.org/10.1146/annurev-physiol-021014-071753

Ito, S., Stuphorn, V., Brown, J. W., \& Schall, J. D. (2003). Performance Monitoring by the Anterior Cingulate Cortex During Saccade Countermanding. Science, 302(5642), 120 122. https://doi.org/10.1126/science. 1087847

Josselyn, S. A., \& Tonegawa, S. (2020). Memory engrams: Recalling the past and imagining the future. Science (New York, N.Y.), 367(6473). https://doi.org/10.1126/science.aaw4325

Kastellakis, G., Cai, D. J., Mednick, S. C., Silva, A. J., \& Poirazi, P. (2015). Synaptic clustering within dendrites: An emerging theory of memory formation. Progress in Neurobiology, 126, 19-35. https://doi.org/10.1016/j.pneurobio.2014.12.002

Kilroy, E., Apostolova, L., Liu, C., Yan, L., Ringman, J., \& Wang, D. J. J. (2014). Reliability of two-dimensional and three-dimensional pseudo-continuous arterial spin labeling 
perfusion MRI in elderly populations: Comparison with 15o-water positron emission tomography: Reliability of 2D Versus 3D pCASL. Journal of Magnetic Resonance Imaging, 39(4), 931-939. https://doi.org/10.1002/jmri.24246

Knudsen, E. I. (2007). Fundamental Components of Attention. Annual Review of Neuroscience, 30(1), 57-78. https://doi.org/10.1146/annurev.neuro.30.051606.094256

Kurth, F., Zilles, K., Fox, P. T., Laird, A. R., \& Eickhoff, S. B. (2010). A link between the systems: Functional differentiation and integration within the human insula revealed by meta-analysis. Brain Structure \& Function, 214(5-6), 519-534. https://doi.org/10.1007/s00429-010-0255-z

Leinweber, M., Ward, D. R., Sobczak, J. M., Attinger, A., \& Keller, G. B. (2017). A Sensorimotor Circuit in Mouse Cortex for Visual Flow Predictions. Neuron, 95(6), 1420-1432.e5. https://doi.org/10.1016/j.neuron.2017.08.036

Lewis, C. M., Baldassarre, A., Committeri, G., Romani, G. L., \& Corbetta, M. (2009). Learning sculpts the spontaneous activity of the resting human brain. Proceedings of the National Academy of Sciences, 106(41), 17558-17563. https://doi.org/10.1073/pnas.0902455106

Li, H. L., \& van Rossum, M. C. (2020). Energy efficient synaptic plasticity. ELife, 9, e50804. https://doi.org/10.7554/eLife.50804

Logothetis, N. K., Pauls, J., Augath, M., Trinath, T., \& Oeltermann, A. (2001). Neurophysiological investigation of the basis of the fMRI signal. Nature, 412(6843), 150-157. https://doi.org/10.1038/35084005

Lundgaard, I., Li, B., Xie, L., Kang, H., Sanggaard, S., Haswell, J. D. R., Sun, W., Goldman, S., Blekot, S., Nielsen, M., Takano, T., Deane, R., \& Nedergaard, M. (2015). Direct neuronal glucose uptake heralds activity-dependent increases in cerebral metabolism. Nature Communications, 6(1), 6807. https://doi.org/10.1038/ncomms7807

Magistretti, P. J., \& Allaman, I. (2015). A Cellular Perspective on Brain Energy Metabolism and Functional Imaging. Neuron, 86(4), 883-901. https://doi.org/10.1016/j.neuron.2015.03.035

Mau, W., Hasselmo, M. E., \& Cai, D. J. (2020). The brain in motion: How ensemble fluidity drives memory-updating and flexibility. ELife, 9, e63550. https://doi.org/10.7554/eLife.63550

Maunsell, J. H. R. (1995). The Brain's Visual World: Representation of Visual Targets in $\begin{array}{llll}\text { Cerebral Cortex. } & \text { 2764-769. }\end{array}$ https://doi.org/10.1126/science.270.5237.764

Menon, V., \& Uddin, L. Q. (2010). Saliency, switching, attention and control: A network model of insula function. Brain Structure \& Function, 214(5-6), 655-667. https://doi.org/10.1007/s00429-010-0262-0

Mergenthaler, P., Lindauer, U., Dienel, G. A., \& Meisel, A. (2013). Sugar for the brain: The role of glucose in physiological and pathological brain function. Trends in Neurosciences, 36(10), 587-597. https://doi.org/10.1016/j.tins.2013.07.001

Miller, E. K., \& Cohen, J. D. (2001). An Integrative Theory of Prefrontal Cortex Function. Annual $\begin{array}{lll}\text { Review of Neuroscience, } & \text { 167-202. }\end{array}$ https://doi.org/10.1146/annurev.neuro.24.1.167

Mishra, A., Reynolds, J. P., Chen, Y., Gourine, A. V., Rusakov, D. A., \& Attwell, D. (2016). Astrocytes mediate neurovascular signaling to capillary pericytes but not to arterioles. Nature Neuroscience, 19(12), 1619-1627. https://doi.org/10.1038/nn.4428

Mohr, H., Wolfensteller, U., Betzel, R. F., Mišić, B., Sporns, O., Richiardi, J., \& Ruge, H. (2016). Integration and segregation of large-scale brain networks during short-term task 
$\begin{array}{llll}\text { automatization. } & \text { Nature } & \text { Communications, } & 7(1),\end{array}$ https://doi.org/10.1038/ncomms13217

Moore, T., \& Zirnsak, M. (2017). Neural Mechanisms of Selective Visual Attention. Annual Review of Psychology, 68(1), 47-72. https://doi.org/10.1146/annurev-psych-122414033400

Mumford, D. (1992). On the computational architecture of the neocortex. Biological Cybernetics, 66(3), 241-251. https://doi.org/10.1007/BF00198477

Oehrn, C. R., Hanslmayr, S., Fell, J., Deuker, L., Kremers, N. A., Do Lam, A. T., Elger, C. E., \& Axmacher, N. (2014). Neural communication patterns underlying conflict detection, resolution, and adaptation. The Journal of Neuroscience: The Official Journal of the Society for Neuroscience, 34(31), 10438-10452. https://doi.org/10.1523/JNEUROSCI.3099-13.2014

Parks, E. L., \& Madden, D. J. (2013). Brain Connectivity and Visual Attention. Brain Connectivity, 3(4), 317-338. https://doi.org/10.1089/brain.2012.0139

Phelps, M. E., Kuhl, D. E., \& Mazziota, J. C. (1981). Metabolic mapping of the brain's response to visual stimulation: Studies in humans. Science (New York, N.Y.), 211(4489), 14451448.

Plaçais, P.-Y., de Tredern, É., Scheunemann, L., Trannoy, S., Goguel, V., Han, K.-A., Isabel, G., \& Preat, T. (2017). Upregulated energy metabolism in the Drosophila mushroom body is the trigger for long-term memory. Nature Communications, 8(1), 15510. https://doi.org/10.1038/ncomms15510

Power, J. D., Schlaggar, B. L., \& Petersen, S. E. (2015). Recent progress and outstanding issues in motion correction in resting state fMRI. Neurolmage, 0, 536-551. https://doi.org/10.1016/j.neuroimage.2014.10.044

Raichle, M. E., \& Mintun, M. A. (2006). Brain Work and Brain Imaging. Annual Review of Neuroscience, 29(1), 449-476. https://doi.org/10.1146/annurev.neuro.29.051605.112819

Redondo, R. L., \& Morris, R. G. M. (2011). Making memories last: The synaptic tagging and capture hypothesis. Nature Reviews Neuroscience, 12(1), 17-30. https://doi.org/10.1038/nrn2963

Reinhart, R. M. G., Zhu, J., Park, S., \& Woodman, G. F. (2015). Synchronizing theta oscillations with direct-current stimulation strengthens adaptive control in the human brain. Proceedings of the National Academy of Sciences, 112(30), 9448-9453. https://doi.org/10.1073/pnas.1504196112

Riedl, V., Utz, L., Castrillón, G., Grimmer, T., Rauschecker, J. P., Ploner, M., Friston, K. J., Drzezga, A., \& Sorg, C. (2016). Metabolic connectivity mapping reveals effective connectivity in the resting human brain. Proceedings of the National Academy of Sciences, 113(2), 428-433. https://doi.org/10.1073/pnas.1513752113

Rischka, L., Godbersen, G. M., Pichler, V., Michenthaler, P., Klug, S., Klöbl, M., Ritter, V., Wadsak, W., Hacker, M., Kasper, S., Lanzenberger, R., \& Hahn, A. (2021). Reliability of task-specific neuronal activation assessed with functional PET, ASL and BOLD imaging. Journal of Cerebral Blood Flow \& Metabolism, 0271678X211020589. https://doi.org/10.1177/0271678X211020589

Rischka, L., Gryglewski, G., Pfaff, S., Vanicek, T., Hienert, M., Klöbl, M., Hartenbach, M., Haug, A., Wadsak, W., Mitterhauser, M., Hacker, M., Kasper, S., Lanzenberger, R., \& Hahn, A. (2018). Reduced task durations in functional PET imaging with [18F]FDG approaching that of functional MRI. Neurolmage, 181, 323-330. https://doi.org/10.1016/j.neuroimage.2018.06.079 
Rogerson, T., Cai, D., Frank, A., Sano, Y., Shobe, J., Aranda, M. L., \& Silva, A. J. (2014). Synaptic Tagging During Memory Allocation. Nature Reviews. Neuroscience, 15(3), 157-169. https://doi.org/10.1038/nrn3667

Sampaio-Baptista, C., Filippini, N., Stagg, C. J., Near, J., Scholz, J., \& Johansen-Berg, H. (2015). Changes in functional connectivity and GABA levels with long-term motor learning. Neurolmage, 106, 15-20. https://doi.org/10.1016/j.neuroimage.2014.11.032

Scholz, J., Klein, M. C., Behrens, T. E. J., \& Johansen-Berg, H. (2009). Training induces changes in white matter architecture. Nature Neuroscience, 12(11), 1370-1371. https://doi.org/10.1038/nn.2412

Shallice, T., Broadbent, D. E., \& Weiskrantz, L. (1982). Specific impairments of planning. Philosophical Transactions of the Royal Society of London. B, Biological Sciences, 298(1089), 199-209. https://doi.org/10.1098/rstb.1982.0082

Shen, C., Ardid, S., Kaping, D., Westendorff, S., Everling, S., \& Womelsdorf, T. (2015). Anterior Cingulate Cortex Cells Identify Process-Specific Errors of Attentional Control Prior to Transient Prefrontal-Cingulate Inhibition. Cerebral Cortex (New York, N.Y.: 1991), 25(8), 2213-2228. https://doi.org/10.1093/cercor/bhu028

Shenhav, A., Botvinick, M. M., \& Cohen, J. D. (2013). The expected value of control: An integrative theory of anterior cingulate cortex function. Neuron, 79(2), 217-240. https://doi.org/10.1016/j.neuron.2013.07.007

Shepard, R. N., \& Metzler, J. (1971). Mental rotation of three-dimensional objects. Science, 171(3972), 701-703. https://doi.org/10.1126/science.171.3972.701

Sheth, S. A., Mian, M. K., Patel, S. R., Asaad, W. F., Williams, Z. M., Dougherty, D. D., Bush, G., \& Eskandar, E. N. (2012). Human dorsal anterior cingulate cortex neurons mediate ongoing behavioural adaptation. Nature, 488(7410), 218-221. https://doi.org/10.1038/nature11239

Smith, S. M., Jenkinson, M., Johansen-Berg, H., Rueckert, D., Nichols, T. E., Mackay, C. E., Watkins, K. E., Ciccarelli, O., Cader, M. Z., Matthews, P. M., \& Behrens, T. E. J. (2006). Tract-based spatial statistics: Voxelwise analysis of multi-subject diffusion data. Neurolmage, 31(4), 1487-1505. https://doi.org/10.1016/j.neuroimage.2006.02.024

Somers, D. C., \& Sheremata, S. L. (2013). Attention maps in the brain. Wiley Interdisciplinary Reviews. Cognitive Science, 4(4), 327-340. https://doi.org/10.1002/wcs.1230

Sridharan, D., Levitin, D. J., \& Menon, V. (2008). A critical role for the right fronto-insular cortex in switching between central-executive and default-mode networks. Proceedings of the National Academy of Sciences, 105(34), 12569-12574. https://doi.org/10.1073/pnas.0800005105

Sun, F. T., Miller, L. M., \& D'Esposito, M. (2004). Measuring interregional functional connectivity using coherence and partial coherence analyses of fMRI data. Neurolmage, 21(2), 647-658. https://doi.org/10.1016/j.neuroimage.2003.09.056

Tang, H., Yu, H.-Y., Chou, C.-C., Crone, N. E., Madsen, J. R., Anderson, W. S., \& Kreiman, G. (2016). Cascade of neural processing orchestrates cognitive control in human frontal cortex. ELife, 5, e12352. https://doi.org/10.7554/eLife.12352

Uddin, L. Q. (2015). Salience processing and insular cortical function and dysfunction. Nature Reviews Neuroscience, 16(1), 55-61. https://doi.org/10.1038/nrn3857

Uddin, L. Q., Supekar, K., Amin, H., Rykhlevskaia, E., Nguyen, D. A., Greicius, M. D., \& Menon, V. (2010). Dissociable Connectivity within Human Angular Gyrus and Intraparietal Sulcus: Evidence from Functional and Structural Connectivity. Cerebral Cortex (New York, NY), 20(11), 2636-2646. https://doi.org/10.1093/cercor/bhq011 
Ullsperger, M., Danielmeier, C., \& Jocham, G. (2014). Neurophysiology of Performance Monitoring and Adaptive Behavior. Physiological Reviews, 94(1), 35-79. https://doi.org/10.1152/physrev.00041.2012

Unterrainer, J. M., Rahm, B., Kaller, C. P., Leonhart, R., Quiske, K., Hoppe-Seyler, K., Meier, C., Müller, C., \& Halsband, U. (2004). Planning Abilities and the Tower of London: Is This Task Measuring a Discrete Cognitive Function? Journal of Clinical and Experimental Neuropsychology, 26(6), 846-856. https://doi.org/10.1080/13803390490509574

Van der Elst, W., Ouwehand, C., van Rijn, P., Lee, N., Van Boxtel, M., \& Jolles, J. (2013). The Shortened Raven Standard Progressive Matrices: Item Response Theory-Based Psychometric Analyses and Normative Data. Assessment, 20(1), 48-59. https://doi.org/10.1177/1073191111415999

Vossel, S., Geng, J. J., \& Fink, G. R. (2014). Dorsal and Ventral Attention Systems. The Neuroscientist, 20(2), 150-159. https://doi.org/10.1177/1073858413494269

Walsh, K. S., McGovern, D. P., Clark, A., \& O'Connell, R. G. (2020). Evaluating the neurophysiological evidence for predictive processing as a model of perception. Annals of the New York Academy of Sciences, 1464(1), 242-268. https://doi.org/10.1111/nyas.14321

Wang, J., Zhang, Y., Wolf, R. L., Roc, A. C., Alsop, D. C., \& Detre, J. A. (2005). Amplitudemodulated Continuous Arterial Spin-labeling 3.0-T Perfusion MR Imaging with a Single Coil: Feasibility Study. Radiology, 235(1), 218-228. https://doi.org/10.1148/radiol.2351031663

Zacks, J. M. (2008). Neuroimaging studies of mental rotation: A meta-analysis and review. Journal of Cognitive Neuroscience, 20(1), 1-19. https://doi.org/10.1162/jocn.2008.20013

Zatorre, R. J., Fields, R. D., \& Johansen-Berg, H. (2012). Plasticity in gray and white: Neuroimaging changes in brain structure during learning. Nature Neuroscience, 15(4), 528-536. https://doi.org/10.1038/nn.3045

Zhang, S., Xu, M., Kamigaki, T., Do, J. P. H., Chang, W.-C., Jenvay, S., Miyamichi, K., Luo, L., \& Dan, Y. (2014). Long-range and local circuits for top-down modulation of visual cortex processing. Science, 345(6197), 660-665. https://doi.org/10.1126/science.1254126

Zimmer, E. R., Parent, M. J., Souza, D. G., Leuzy, A., Lecrux, C., Kim, H.-I., Gauthier, S., Pellerin, L., Hamel, E., \& Rosa-Neto, P. (2017). [18F]FDG PET signal is driven by astroglial glutamate transport. Nature Neuroscience, 20(3), 393-395. https://doi.org/10.1038/nn.4492 


\section{FIGURES}

a) Design

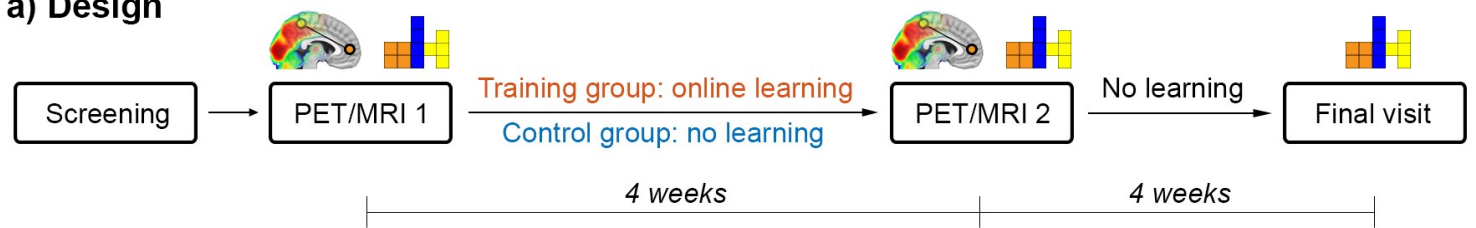

b) Energy demands

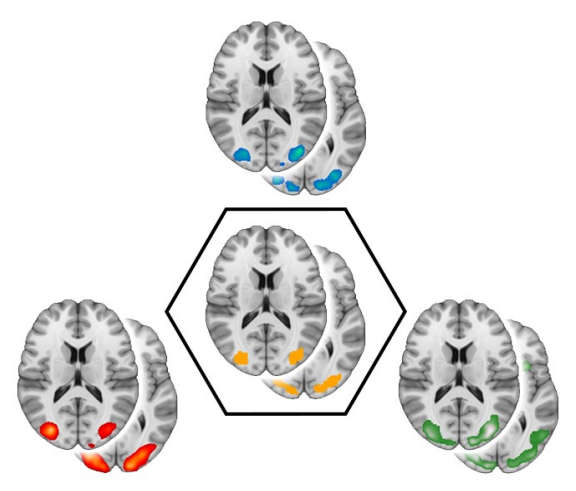

c) Network analysis

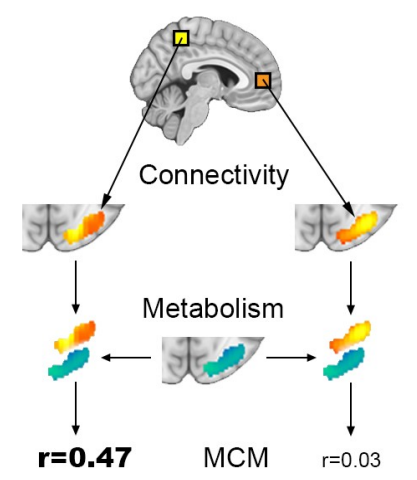

d) Simulations

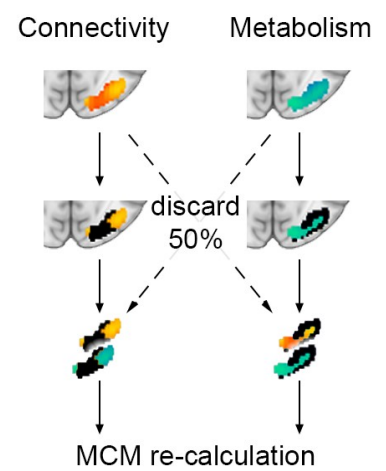

Figure 1: Design and analysis. a) After the initial screening, participants were randomly assigned to the training or the passive control group. All subjects underwent two simultaneous PET/MRI examinations for acquisition of structural, functional and metabolic data at restingstate and while performing a challenging visuo-spatial processing task (the video game Tetris $®$, supplementary figure S1). In the 4-week period between the two PET/MRI scans, the training group regularly practiced the task using an online platform, whereas the control group did not. After the second PET/MRI scan, no further training was carried out and the training group completed a final task session on a laptop. Additional testing of different cognitive domains was performed at both PET/MRI examinations. b) To obtain a robust estimate of taskspecific increases in energy demands, the imaging parameters of glucose metabolism (blue), cerebral blood flow (green) and BOLD-derived activation (red) were combined in a conjunction analysis (intersection, orange). Joint active areas served as target regions for the subsequent network analysis (supplementary figure S2). c) We extended metabolic connectivity mapping (MCM) to the whole brain level to assess learning-induced adaptations in directional connectivity towards regions with high task-specific energy demands. The BOLD signal of each 
brain voxel (exemplarily shown as yellow/orange squares) yields a certain functional connectivity pattern in the target region (here the occipital cortex). Computing the spatial correlation between patterns of functional connectivity (yellow/orange) and glucose metabolism (blue/green) results in an MCM value for each brain voxel that reflects the directional connectivity to the target. d) Finally, simulations were carried out to disentangle the individual contribution of glucose metabolism and functional connectivity to MCM learning effects. Voxels in the target region were gradually removed based on values of connectivity or metabolism (here $50 \%$ black voxels in left and right columns, respectively), followed by recalculation of MCM values and the corresponding learning effects. 

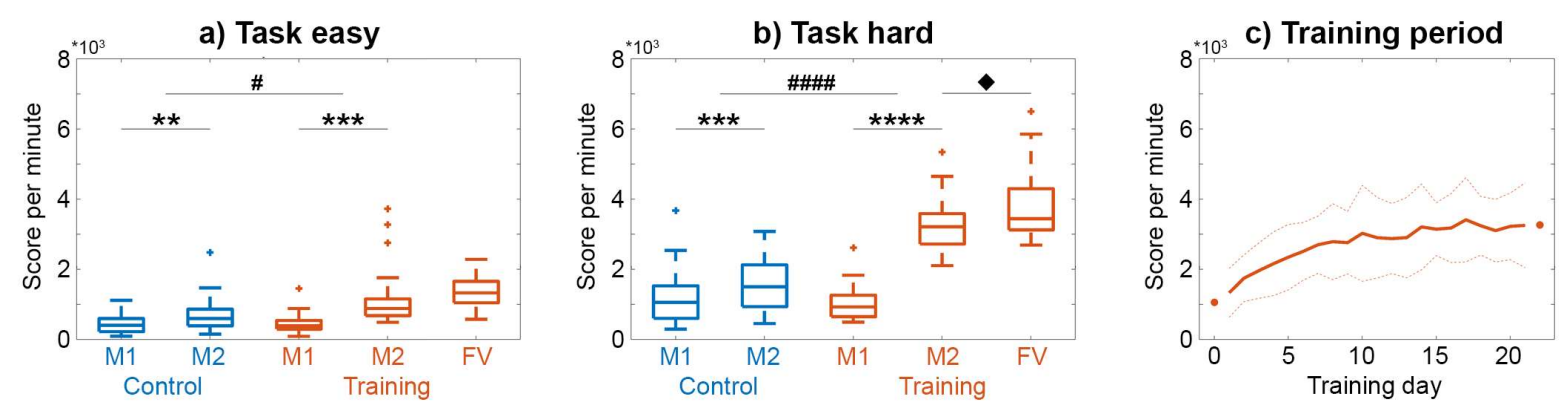

Figure 2: Behavioral data for the video game Tetris ${ }^{\circ}$ measured as score per minute. Changes in task performance differed between the two PET/MRI measurements, groups and task conditions (group*time*condition interaction, $p<10^{-5}$ ). a) For the easy task condition, the training group showed a 2.7-fold increase in performance, which was significantly higher compared to the control group. b) For the hard task, changes in performance followed a similar pattern but effects were more pronounced, with the training group showing a 3.1-fold improvement in performance. Also, task performance for the hard task condition further increased even without training until the final visit (FV). The time between measurements/visits was 4 weeks. Initial performance at measurement 1 was not significantly different between the groups for both task conditions ( $p>0.5)$. c) Monitoring the task performance during the training period highlights the continuous improvement. The learning curve further matched with the performance of the two PET/MRI measurements as indicated by the dots (average values of M1 and M2 in b). Solid and dotted lines represent mean and standard deviation, respectively. Data were cut after 21 days as less than $1 / 3$ of the subjects trained longer than this period. For $\mathbf{a}$ and $\mathbf{b}$, post-hoc comparisons indicate significant differences for the group*time interactions

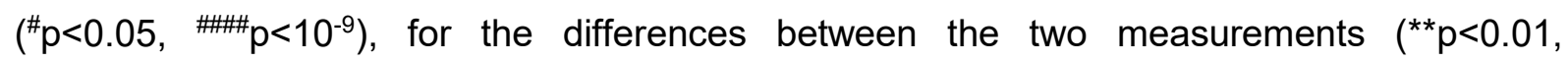
$\left.{ }^{* * *} p<0.001,{ }^{* * *} p<10^{-10}\right)$ and for the difference between measurement 2 and the final visit $\left({ }^{\star} p 0.05\right)$. All $p$-values were corrected for multiple comparisons with the Bonferroni-Holm procedure. 
a)

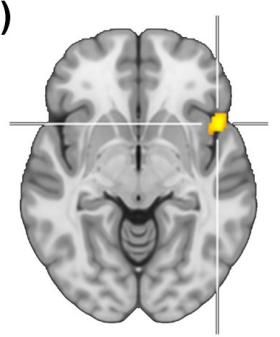

b)

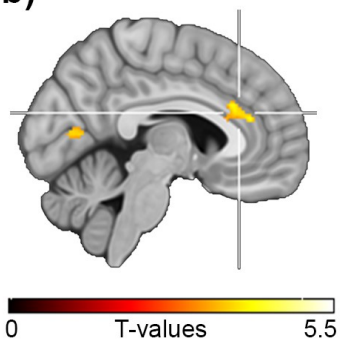

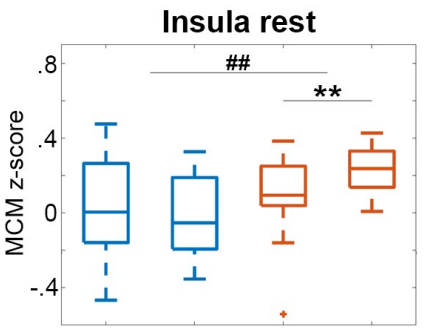

dACC rest

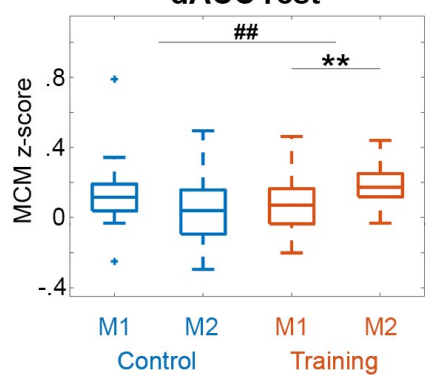

Insula task easy

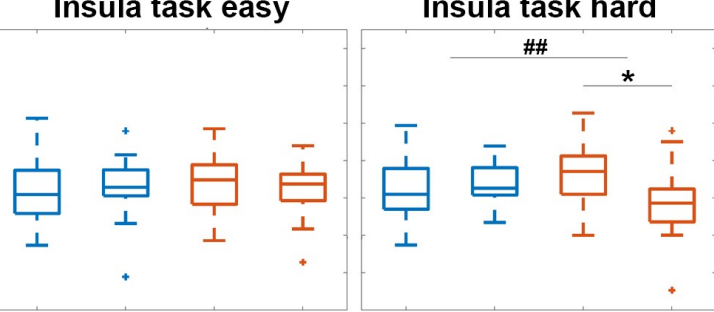

dACC task easy

dACC task hard

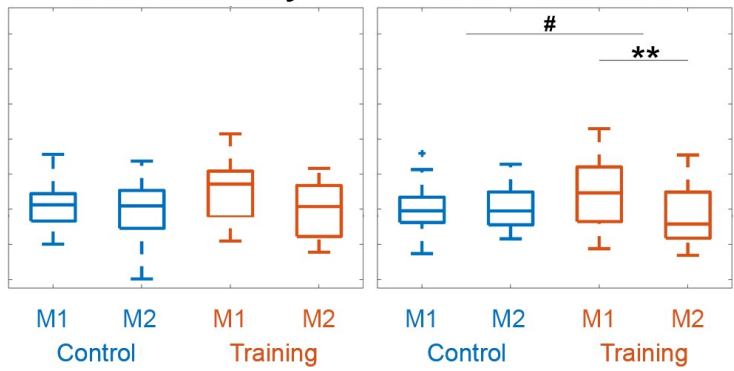

Figure 3: Learning-induced changes in metabolic connectivity mapping (MCM) with the occipital cortex as target region. Four weeks of training the video game Tetris $₫$ resulted in specific adaptations of connectivity from the right insula (a) and the dorsal anterior cingulate cortex $\left(\mathrm{dACC}\right.$, b) to the occipital cortex $\left(\right.$ group*time ${ }^{*}$ condition interaction, $p<0.05$ FWEcorrected cluster level). Post-hoc comparisons showed that at rest MCM increased for both connections in the training group as compared to the control group. In contrast, MCM decreased during the hard task condition in the training group. There were no significant changes in the control group between the two measurements. Furthermore, MCM values between training and control groups at measurement 1 were not significantly different. Boxplots show the MCM z-scores of the clusters indicated by the crosshair. Post-hoc comparisons indicate significant differences for the group ${ }^{*}$ time interaction $\left({ }^{\#} p<0.05,{ }^{\#} p<0.01\right)$ and for the differences between the two measurements $\left({ }^{*} p<0.05,{ }^{* *} p<0.01\right)$, corrected for multiple comparisons with the Bonferroni-Holm procedure. 
a) Tetris ${ }^{\circledR}$ PET/MRI 2

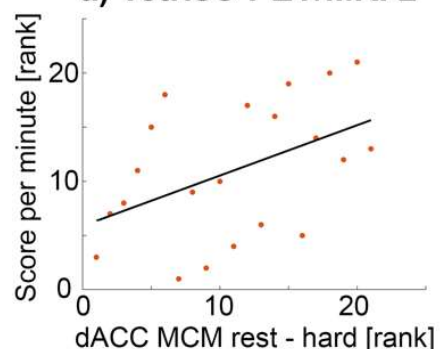

b) Tetris $₫$ training

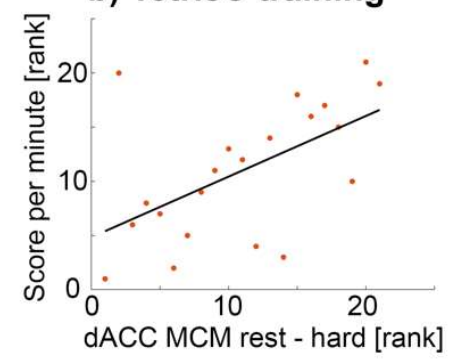

c) Mental rotation

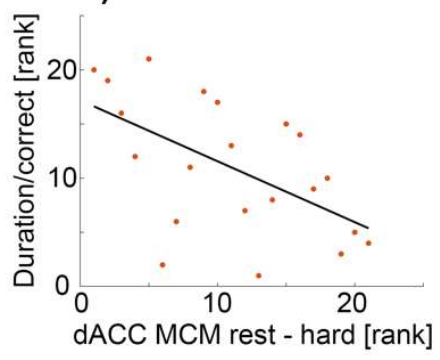

Figure 4: Associations between MCM adaptations and cognitive performance. Based on the training-induced effects in the salience network (figure 3), one would expect that subjects with high MCM values at rest and low values during task execution (i.e., a high divergence between rest and task) show the best cognitive performance after learning. Thus, the difference of MCM values between rest and the hard task condition at the second PET/MRI scan was correlated with task performance. Positive associations of $\mathrm{dACC}$ MCM values were observed with the Tetris ${ }^{\circledR}$ score (high score $=$ high performance) of the second PET/MRI measurement $(\mathbf{a}$, rho $=0.46, p<0.05)$ and that obtained during the 4-week training period $(\mathbf{b}$, rho $=0.56, p<0.01$, normalized area under curve). Further, dACC MCM values were negatively associated with the mental rotation performance (duration/number of correct answers with low value $=$ high performance, $\mathbf{c}$, rho $=-0.56, \mathrm{p}<0.01)$. All values were rank transformed to account for one outlier, thus correlation values represent Spearman's rho. 

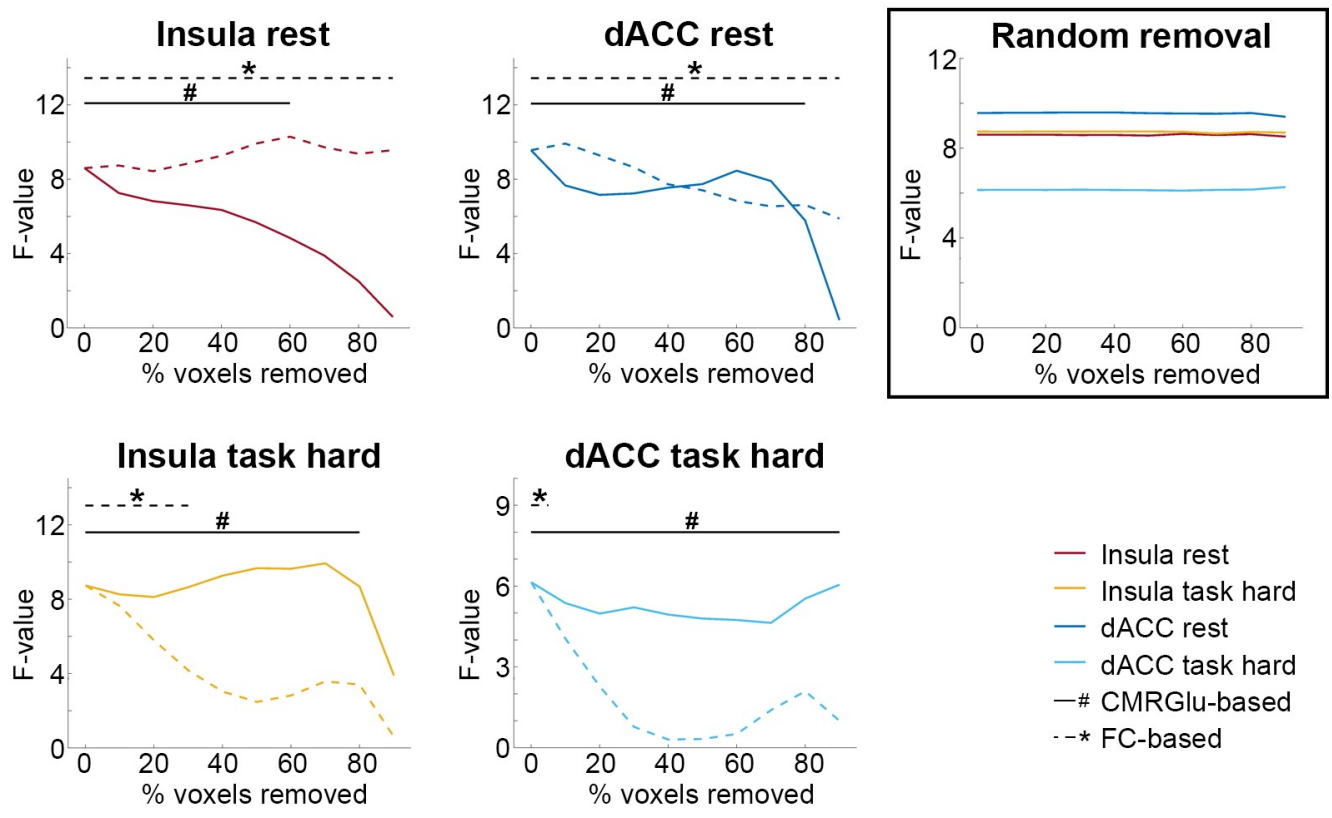

Figure 5: Simulated perturbations of learning-induced changes in metabolic connectivity mapping (MCM). We aimed to identify whether learning-specific MCM effects in figure 3 were driven by glucose metabolism (CMRGlu) or functional connectivity (FC). Voxels of the occipital cortex (i.e., the MCM target region) were progressively removed based on increasing values of CMRGlu (solid lines) or FC (dashed lines) and training effects were re-calculated (F-value of group*time interaction). At resting-state simulated removal of voxels based on CMRGlu abolished training-induced MCM effects for both connections towards the occipital cortex, which was, however, not the case for FC (top row). The inverse pattern was observed for the hard task condition, where training-specific decreases in MCM were nullified when removing voxels based on FC, but not CMRGlu (bottom row). \#/solid black lines: $p<0.05$ when removing voxels based on CMRGlu. */dashed back lines: $p<0.05$ when removing voxels based on FC values. $0 \%$ of voxels removed represents the results shown in figure 3 (i.e., when using the entire target region). Of note, randomly removing up to $90 \%$ of voxels in the occipital cortex did not affect the learning-induced changes in MCM at all (right bottom panel, all $p<0.05$ ), highlighting the specificity of CMRGlu and FC to drive MCM changes and indicating that effects are not dependent on the size of the target region. The colors for the random removal match those of the other panels. 


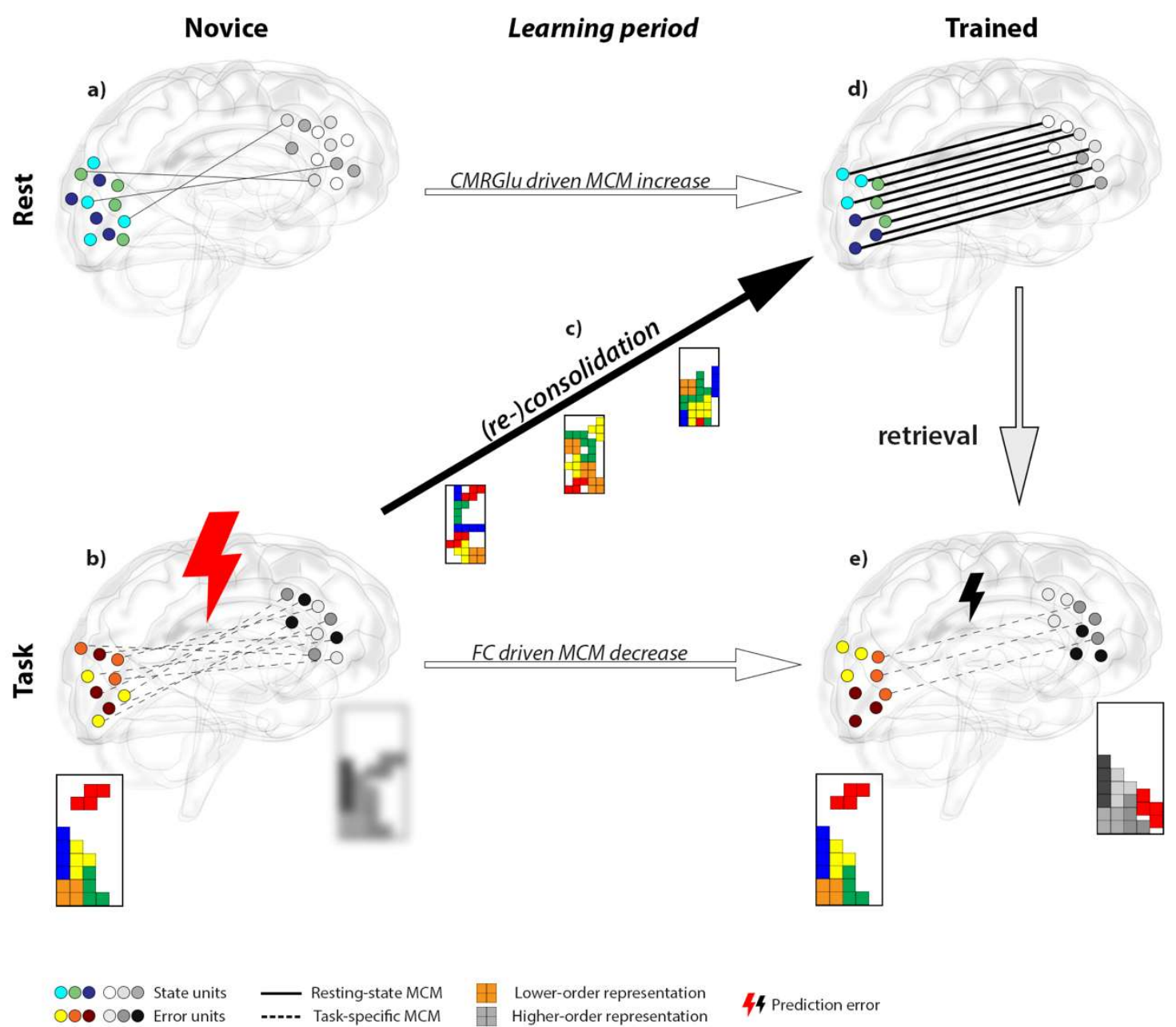

Figure 6: Schematic illustration of training effects and potential neurobiological mechanisms. Cognitive skill learning results in complementary metabolic adaptations at rest (top row) and functional network reorganization during task execution (bottom row). Glass brains depict directional connectivity from the higher-order salience network (grayscale circles) to the lowerorder occipital region (colored circles) as assessed with metabolic connectivity mapping (MCM, number of lines). a) Training naïve subjects exhibit low directional connectivity at resting-state (solid lines) between unorganized state units (blue-green circles), since the skill trace is not yet established (random circle arrangement, crossing lines). b) Due to the lack of training, higher-order representations in the $\mathrm{SN}$ are inaccurate (blurred grayscale Tetris $\circledR$ ) in comparison to lower-order visual sensory information (colored Tetris $®$ ), resulting in a high prediction error (large thunderbolt) encoded by error units (red-yellow circles). The representational inaccuracy requires substantial dynamic optimization between brain regions 
of different hierarchies (numerous dashed lines). c) With repeated task performance during the learning period functional network reorganization approaches an optimal solution. Presumably, this is realized by a high frequency of synaptic tagging, where optimal task representations are gradually encoded in the salience network by synaptic capture and subsequent anchoring of glutamatergic AMPA receptors. d) After the learning period, state unit directional connectivity increases, which equals the consolidated skill engram (parallel lines between organized circles). The metabolic emphasis of this process suggests the energyintensive formation of clustered and potentiated synapses (line thickness). e) The established skill engram can then be retrieved for task execution. This results in a decreased prediction error (small thunderbolt) as representations between higher- and lower-order brain regions became more accurate (sharpened grayscale Tetris ${ }^{\circledR}$ ). Thus, only minor cognitive control is required (few dashed lines) to apply an efficient task strategy. In sum, these observations indicate that effects of skill learning at resting-state and during task execution are two sides of the same coin, where different neurobiological mechanisms complement each other to improve task performance. The glass brain was kindly provided by Dr. Gill Brown (https://neurosciencegraphicdesign.com/) under CC BY-NC 4.0. 


\section{Supplementary Information for}

\section{Learning induces coordinated neuronal plasticity of metabolic demands and functional brain networks}

Sebastian Klugi", Godber M Godbersen ${ }^{1 \#, ~ L u c a s ~ R i s c h k a 1, ~ W o l f g a n g ~ W a d s a k ~}{ }^{2,3}$, Verena Pichler $^{2,4}$, Manfred Klöbl ${ }^{1}$, Marcus Hacker ${ }^{2}$, Rupert Lanzenberger ${ }^{1}$, Andreas Hahn ${ }^{1 *}$

${ }^{1}$ Department of Psychiatry and Psychotherapy, Medical University of Vienna, Austria

${ }^{2}$ Department of Biomedical Imaging and Image-guided Therapy, Division of Nuclear Medicine, Medical University of Vienna, Austria

${ }^{3}$ Center for Biomarker Research in Medicine (CBmed), Graz, Austria

${ }^{4}$ Department of Pharmaceutical Sciences, Division of Pharmaceutical Chemistry, University of Vienna, Austria 


\section{Supplementary Figures}

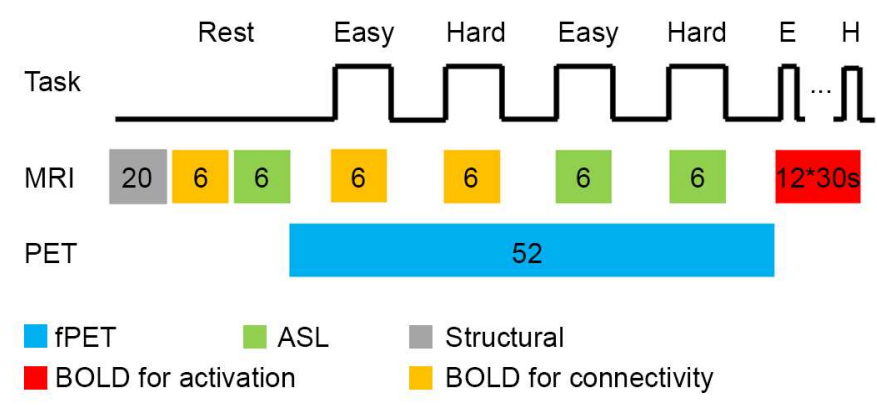

Supplementary figure S1: PET/MRI measurement procedure. Data acquisition included a T1weighted structural scan (gray, $8 \mathrm{~min}$ ) as well as BOLD imaging (orange, $6 \mathrm{~min}$ ) and ASL (green, $6 \mathrm{~min}$ ) at resting state. After that, $\left[{ }^{18} \mathrm{~F}\right] \mathrm{FDG}$ fPET imaging started (blue, $52 \mathrm{~min}$ ) with simultaneous recording of BOLD (orange) and ASL (green). During these MRI sequences, continuous task performance was carried out at two levels of difficulty (easy and hard in randomized order). BOLD sequences obtained at rest and during the task (orange) were used to estimate functional connectivity and subsequently for metabolic connectivity mapping. ASL and $\mathrm{fPET}$ were used to quantify indices of task-induced neuronal activation as CBF and CMRGlu, respectively. After fPET was completed, the last MRI acquisition again comprised BOLD imaging. Here, a conventional block design was employed as a third estimate of taskspecific neuronal activation (red, 12 randomized blocks of easy, hard and control conditions, $30 \mathrm{~s}$ each with $10 \mathrm{~s}$ baseline in-between, total $8.17 \mathrm{~min})$. Numbers indicate measurement time in minutes unless indicated otherwise. This figure was adapted from (Hahn et al., 2020) under the CC BY 4.0 license. 
a) CMRGIu
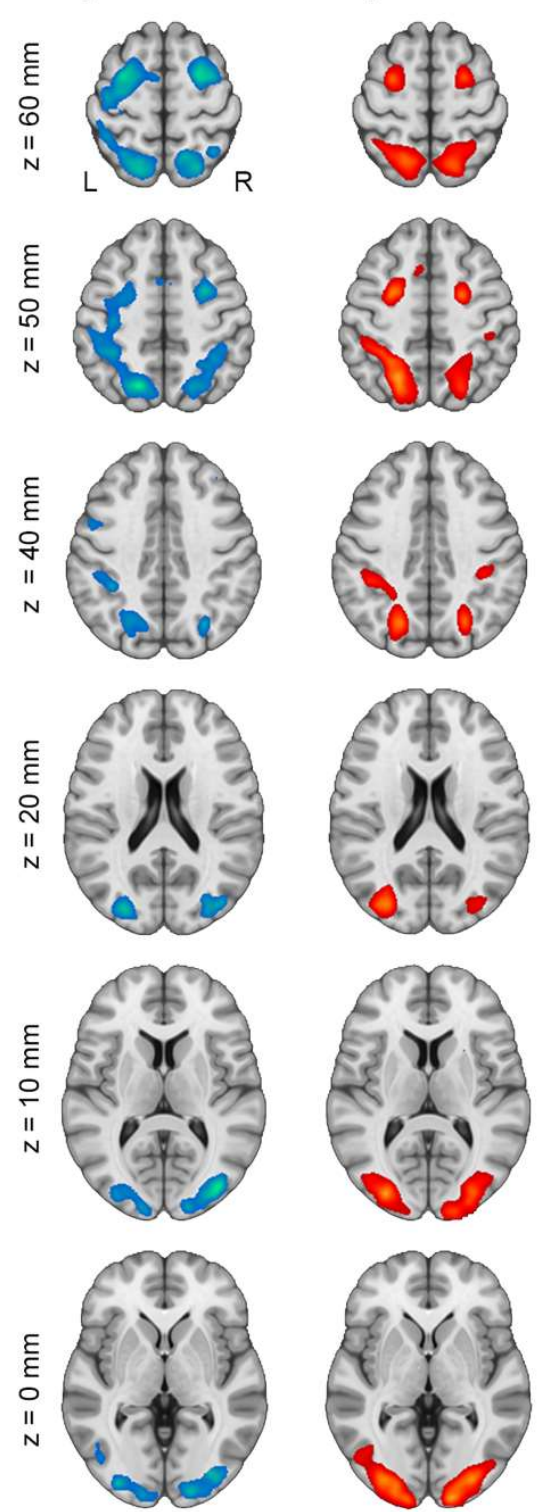

0 T-value \pm 15

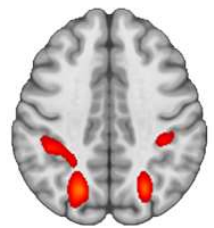

b) BOLD
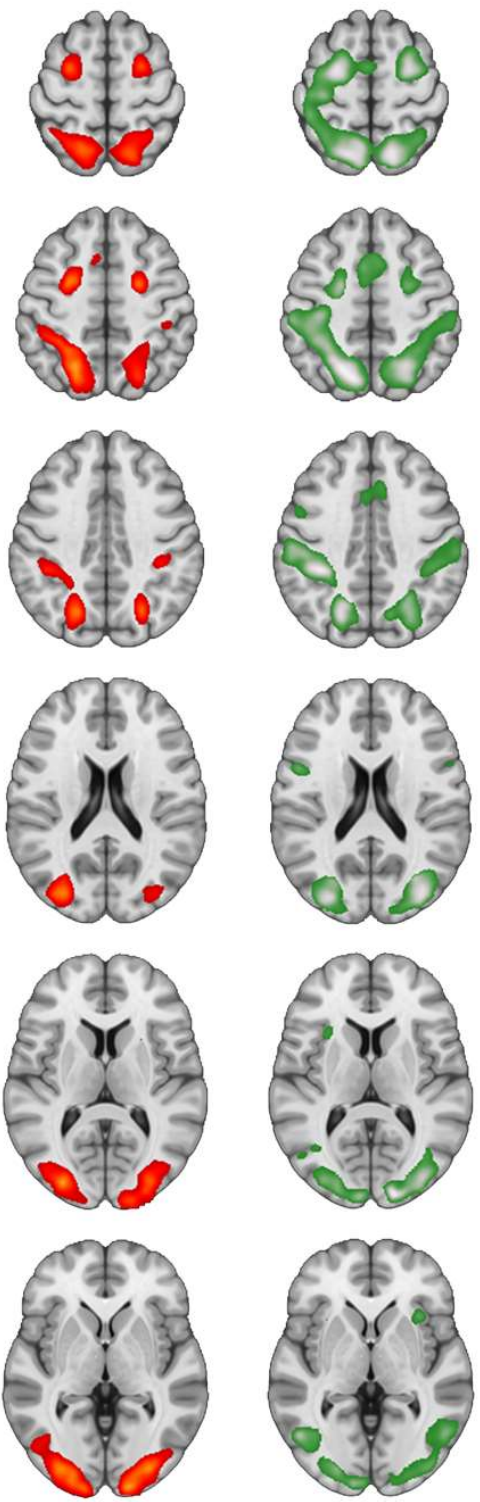

T-value \pm 15 d) Conjunction
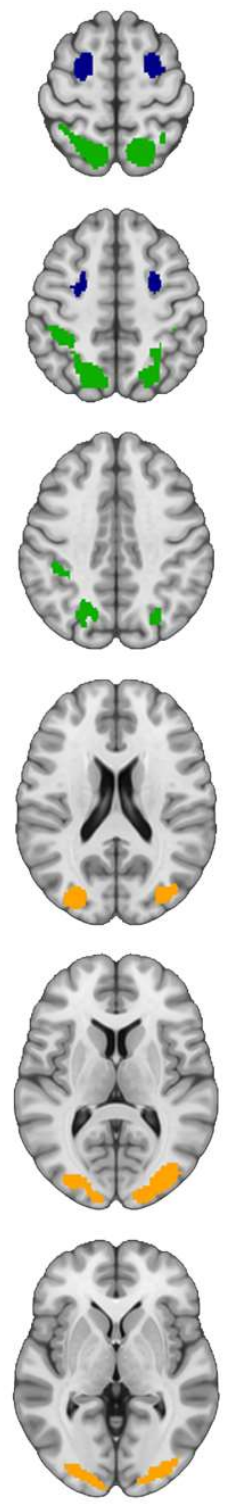

Supplementary figure S2: Multimodal parameters of neuronal activation. Task-induced changes as identified by imaging of the cerebral metabolic rate of glucose (CMRGlu, a), bloodoxygen level dependent signal (BOLD, b) and cerebral blood flow (CBF, c). Group maps were derived from the first PET/MRI measurement $(n=39)$ and thresholded at $p<0.05$ FWE-corrected voxel level. Dice coefficients were 0.57 (CMRGlu vs BOLD), 0.51 (CMRGlu vs CBF) and 0.48 (CBF vs BOLD). The conjunction map (d) shows the spatial overlap in metabolic demands as computed by the intersection across the three imaging modalities (orange: occipital cortex, 
bioRxiv preprint doi: https://doi org/10.1101/2021.11.12468351; this version posted November 12,2021 . The copyright holder for this preprint (which was not certified by peer review) is the author/funder, who has granted bioRxiv a license to display the preprint in perpetuity. It is made available under aCC-BY-NC-ND 4.0 International license.

green: intraparietal sulcus, blue: frontal eye field). Those regions showing an overlap in taskspecific activation were used as target regions for the subsequent metabolic connectivity mapping (MCM) analysis. Axial slices are shown in neurological convention (left is left). 